\title{
LA PRESIDENZA DELLA COMMISSIONE EUROPEA. VINCOLI E RISORSE
}

\author{
di Ken Endo
}

\section{Introduzione}

La carica di Presidente della Commissione Europea è malleabile. I suoi poteri e le sue possibilità di incidenza politica mutano con l'avvicendarsi dei presidenti, e anche nel corso di una stessa presidenza, come mostra il caso di Jacques Delors, il cui ufficio è passato in un decennio (1985-1995) attraverso fasi espansive e restrittive.

La «malleabilità» della Presidenza della Commissione può essere in parte attribuita al fatto che la carica è ancora giovane, con solo 35 anni di storia. Peraltro, la stessa Unione Europea è ben lontana dall'aver raggiunto la sua configurazione definitiva. Storicamente, la natura dell'ufficio è stata influenzata da due fattori: uno situazionale, l'altro personale. Nel caso in cui, per esempio, la costellazione dei leader degli stati membri non coopera con la Presidenza, o addirittura manifesta ostilità nei suoi confronti, il detentore della carica è costretto ad adottare un approccio sommesso. Le presidenze di Jean Rey e (finora) di Jacques Santer possono servire come esempi di questo stile più pacato, dopo i decenni delle presidenze particolarmente «espansive» dei loro rispettivi predecessori, Walter Hallstein e Jacques Delors. L'importanza del fattore situazionale emerge anche nei momenti di crisi, presunte o reali, in cui il Presidente della Commissione può avere maggiori opportunità di intervenire politicamente che non in situazioni normali. Il modo in cui la carica di Presidente è gestita dipende infine dalle personalità dei singoli presidenti. Per esempio, François-Xavier Ortoli non esercitò forti pressioni per essere presente ai summit economici delle potenze occidentali, il che contrasta fortemente con la linea del suo successore Roy Jenkins, il quale invece vide nella partecipazione a tali incontri al vertice un'eccellente possibilità 
di migliorare la posizione della Presidenza. Gaston Thorn, poi, non fece pienamente uso della possibilità di partecipare al Consiglio Europeo durante i suoi quattro anni di presidenza, al contrario di Delors, suo successore.

Ad ogni modo, la natura malleabile della Presidenza della Commissione non significa che non esistano caratteristiche costanti della carica. Al contrario, esiste una struttura istituzionale di base che delimita marcatamente l'ambito in cui il Presidente della Commissione può esercitare la sua leadership. Da un lato, nessun leader è in grado di esercitare una leadership politica senza risorse da cui trarre potere, anche se le circostanze in cui si trova ad operare gli offrissero molte opportunità o se possedesse un grande talento e spiccate capacità. Dall'altro, nessun leader dispone di un potere privo di vincoli. Le risorse di potere ed i vincoli allo stesso concorrono nel segnare dei confini, non necessariamente fissi nel tempo, all'interno dei quali un leader esercita la sua leadership politica. Il Presidente della Commissione non costituisce una eccezione a questa regola: per studiare la sua leadership, dunque, si devono analizzare i confini della sua influenza costituiti dalle risorse e dai vincoli propri del suo ufficio. $\grave{E}$ alla comprensione di questi aspetti che questo articolo tenta di contribuire'.

Il vincolo più grande alla leadership del Presidente della Commissione risiede nella natura policentrica ed altamente frammentata della Comunità Europea. La questione della segmentazione politica della Comunità e della dispersione delle fonti di potere nel suo àmbito non sarà affrontata direttamente. Questo articolo, pur toccando alcune questioni ad essa legate (ad esempio la mancanza di una solida base sociale per la Comunità Europea), si concentrerà invece sul più stretto insieme di disposizioni istituzionali riguardanti la Presidenza della Commissione $o$ ad essa immediatamente connesse. Nei paragrafi che seguono ci si soffermerà dunque principalmente sulla procedu-

1 Questo studio è parte di una più ampia ricerca di dottorato sulla leadership politica del Presidente della Commissione condotta a Oxford. L'autore ha un grande debito di gratitudine verso W. Wallace per la sua supervisione dell'intero studio, e verso V. Wright e R. Morgan per la loro supervisione informale e supplementare. L'autore ringrazia anche L. Bardi, S. Bartolini, J. Blondel, J. Catherall, J. Cloos, R. Corbett, J. Hayward, A. Menon, Y. Mény, K. Nakamura, H. Paemen, G. Peters, M. Vanden Abeele, L. Van Depoele, J. Vignon per i loro suggerimenti ed incoraggiamenti, e infine, i circa 100 funzionari e politici (inclusi tre Presidenti di Commissione) che hanno gentilmente accettato di essere intervistati. 
ra di nomina della Presidenza, sui rapporti del Presidente con il Consiglio, il Parlamento e gli altri Commissari, sugli organi interni della Commissione che sono sotto il controllo del Presidente, su alcune risorse esterne del potere presidenziale, su alcuni vincoli che il Presidente può incontrare nella sua azione politica esterna.

Nell'analisi si utilizzerà principalmente un approccio istituzionalista e comparato (King 1975; Jones 1991a; Hayward 1993; Blondel 1987; Blondel e Müller-Rommel 1993). Va però subito precisato che in questa sede per comparazione non si intende una analisi approfondita che tratti altri esecutivi e altri sistemi politici con la stessa accuratezza del soggetto principale, quanto riferimenti abbastanza occasionali ad altri oggetti che possano contribuire a delineare il profilo delle caratteristiche istituzionali della Presidenza della Commissione. Per quanto riguarda gli oggetti della comparazione, non si è ritenuto opportuno comparare la Presidenza della Commissione con il Segretariato Generale delle Nazioni Unite o la Direzione Generale del GATT, dato che il potere della prima è molto maggiore di quello di questi organismi internazionali. Piuttosto, si sono presi in considerazione $i$ vertici di altri sistemi politici, in particolare di sistemi (con-)federali o consociativi, come la Svizzera e l'Olanda.

\section{Le tre forme della leadership del Presidente della Commissione}

Ci sono almeno tre forme importanti della leadership del Presidente della Commissione: a) la leadership amministrativa; b) la leadership mediatrice; c) la leadership sull'agenda politica (agenda-setting leadership) ${ }^{2}$.

Dal punto di vista storico l'amministrazione centrale della Comunità è stata costruita da zero. La creazione di una struttura amministrativa è un compito molto impegnativo. Fondare un'amministrazione europea - e quindi internazionale - fornita di funzioni esecutive era un compito senza precedenti (Morgan 1992, 7), che avrebbe richiesto una forte leadership. Si possono richiamare, a titolo di esempio, le condizioni in cui si sono trovati i primi vertici dell'Alta Autorità della CECA e le Commis-

\footnotetext{
2 Per un riferimento classico al ruolo e alle funzioni della Commissione, vedi Coombes (1970).
} 
sioni della CEE e dell'Euratom. Jean Monnet - primo Presidente dell'Alta Autorità - ha svolto un ruolo significativo nel reclutare funzionari selezionati con competenze tecniche e (insieme a Pierre Uri - il suo più stretto consigliere) nel disegnare la struttura amministrativa della Comunità Europea del Carbone e dell'Acciaio (Ibid., 3). Walter Hallstein - primo Presidente della Commissione CEE - contribuì in maniera egualmente energica alla creazione della nuova Commissione, reclutando rapidamente circa mille funzionari nel 1958, quando il Consiglio Europeo e il Consiglio dei Rappresentanti Permanenti (COREPER) non avevano ancora cominciato a lavorare a pieno regime (Ivi, 1-6; Nöel 1992, 145 e ss.).

Il tipo di leadership richiesto per realizzare la costruzione di un'amministrazione di questa natura è del tutto peculiare. È una leadership che gestisce direttamente la creazione e l'ammodernamento di un apparato amministrativo centrale responsabile per l'intera sintesi politica.

Una volta che l'amministrazione è stata creata e consolidata, la necessità di questa dimensione amministrativa della leadership diminuisce. Ciò sposta il baricentro della leadership del Presidente della Commissione senza però cancellarne la dimensione amministrativa. Lo sviluppo della Comunità, l'ingresso di nuovi stati membri, la crescita delle dimensioni e dell'articolazione dell'amministrazione, e l'attribuzione ad essa di un numero crescente di funzioni hanno infatti posto nuovi problemi di gestione amministrativa. Il Presidente Roy Jenkins, ad esempio, commissionò il Rapporto Spierenburg nel 1979 nella speranza di poter snellire l'apparato della Commissione, anche se il tentativo rimase poi senza esito. L'attuale Presidente Jacques Santer ha collocato fra le sue priorità il compito di «sollevare il morale dei funzionari della Commissione». La necessità di gestire $o$ anche ristrutturare l'amministrazione e, quindi, di continuare ad esercitare una leadership amministrativa, può dunque protrarsi nel tempo.

La Comunità Europea è una polity profondamente frammentata lungo le linee tracciate dalle nazionalità. Il modello più vicino a questa realtà può essere rinvenuto in un regime confederale o consociativo in cui molti valori e domande confliggenti devono essere composti e in cui il potere è largamente disperso (W. Wallace 1983; Forsyth 1981; Taylor 1993). In un tale modello, se i leader del sistema non riescono a raggiungere i compromessi necessari, il processo di policy-making si blocca e si 
pone in questione l'esistenza stessa della sintesi politica. L'esistenza di stati nazionali resilienti, con una lunga tradizione storica, rende particolarmente evidenti, nel caso della $\mathrm{CE}$, le caratteristiche di un sistema fortemente policentrico. Perché la Comunità possa funzionare è dunque necessario raggiungere dei compromessi. E qui si crea lo spazio per la leadership nella gestione e composizione dei conflitti, cioè per la leadership mediatrice.

Il Presidente della Commissione è in buona posizione per ritagliarsi un proprio ruolo negli accordi e nei compromessi fra leader governativi e altre élites. Innanzitutto, la Commissione non è vista solamente come un organo «passivo», relativamente imparziale nei confronti degli stati membri, ma può anche, attivamente, «far progredire l'interesse comune», per seguire la vecchia formula di Ernst Haas $(1958,133)$. In secondo luogo, il Presidente della Commissione è formalmente membro del Consiglio Europeo, l'organo che riunisce i soggetti che hanno il maggiore potere decisionale nella Comunità, e può partecipare alle riunioni del Consiglio Affari Generali dei Ministri degli Esteri e del Consiglio dei Ministri Economici e Finanziari (Ecofin), i due importanti organi decisionali della CE. In terzo luogo, il Presidente della Commissione è parte integrante del dialogo interistituzionale fra Commissione, Parlamento, e Consiglio Europeo. Dunque, quando vi sono conflitti, il Presidente della Commissione ha diversi canali utili per la ricerca di compromessi.

Un esempio importante dell'esercizio di una leadership mediatrice può essere visto nell'azione politica del Presidente Rey. Attraverso una pacata ma abile azione diplomatica, ha portato a termine il negoziato rimasto incompiuto, volto ad unificare i tre «esecutivi» della Comunità, superando così i problemi di coordinamento ed inefficienza derivanti dalla separazione fra le tre amministrazioni. Il Presidente Delors sembra aver esercitato una leadership mediatrice in diverse occasioni, in particolare nel processo sfociato nell'adozione delle «prospettive finanziarie» (bilanci programmatici) del 1988 e del 1992, e nell'ambito dell'attività del cosiddetto Comitato Delors sull'Unione Economica e Monetaria (Grant 1994).

Rispetto al momento della sua creazione, l'ambiente esterno della Commissione è cambiato. Nei primi anni Ottanta, Helen Wallace ha sostenuto che, una volta conclusa la fase di realizzazione degli obiettivi e di attuazione delle disposizioni dei Tratta- 
ti, il ruolo della Commissione si è politicizzato poiché il contesto imponeva di ridefinire le modalità di intervento politico e di aggregazione degli interessi $(\mathrm{H}$. Wallace 1983, 58). Su diversi fronti la Comunità iniziò infatti ad inoltrarsi in un terreno inesplorato, o «grigio», che il Trattato di Roma non aveva regolato o su cui non aveva dettato chiare disposizioni. Quando si tratta di fissare delle priorità per le diverse questioni e di chiarire in tal modo ciò che la Comunità dovrà fare in futuro, la leadership amministrativa e quella mediatrice non sono sufficienti e si manifesta la necessità di una forma di leadership adatta a questa funzione.

Più efficacemente e rapidamente della Commissione a queste nuove esigenze hanno risposto i leader dei governi degli stati membri. Essi hanno organizzato la loro struttura di leadership nella forma di incontri al vertice, a partire dal 1969. Dalla metà degli anni Settanta i loro summit si sono istituzionalizzati come organo di formazione dell'agenda politica della Comunità, evolvendosi nel Consiglio Europeo e riuscendo ad influenzare così il processo di integrazione. Di pari passo, l'«asse» franco-tedesco è emerso come la vera forza in grado di dettare la rotta della CE e di guidare la politica comunitaria, come l'istituzione del Consiglio Europeo sta a dimostrare. Dalla metà degli anni Sessanta, inoltre, la Presidenza del Consiglio Europeo, detenuta a rotazione dai governi degli stati membri, è divenuta sempre più influente nella formazione dell'agenda politica della Comunità. Così, man mano che la Comunità cominciava ad intervenire nell'area non esplicitamente governata dal Trattato, i governi degli stati membri e i loro leader sono stati fortemente coinvolti nella formazione dell'agenda politica della Comunità.

Ciò nonostante, alla Commissione, e in particolare al suo presidente, non è stato negato un ruolo di leadership nella strategia della definizione dell'agenda politica. Il presidente Jenkins, ad esempio, si batté duramente per rilanciare l'attenzione sulla questione dell'unione monetaria e per metterla all'ordine del giorno della politica comunitaria; la questione sarebbe poi stata portata avanti con efficacia dal Cancelliere tedesco Helmut Schmidt (Ludlow 1982). Il vicepresidente Etienne Davignon, personaggio dominante nella Commissione Thorn, è stato una figura centrale nel lancio di ESPRIT (Programma Strategico Europeo Ricerca e Sviluppo Tecnologico dell'Informazione). Questo nuovo schema industriale e tecnologico ha contribuito ad alimentare la cooperazione fra gli industriali europei, il che si sarebbe poi rivelato di importanza cruciale per il 
successo del programma del Mercato Interno (Sharp 1990). Infine, Delors ha avuto spesso un ruolo importante nel determinare la rotta politica della Comunità: durante le trattative per l'Atto Unico Europeo, nella definizione del pacchetto Delors I, nella formazione del piano per l'unione monetaria, e per il varo del Libro Bianco del 1993 (Grant 1994; Ross 1995).

Le vicende citate indicano chiaramente che è possibile per la Commissione, e in particolare per il suo Presidente, avere una parte nell'individuazione di mete strategiche per la Comunità. Quando si tratta di costruire la Comunità al di là dell'ambito originariamente previsto nei Trattati, e quindi in aree normalmente gestite dai leader dei governi nazionali, al Presidente della Commissione si richiede di interpretare l'interesse generale e il punto di vista dell'Europa. Egli dovrà pertanto difendere davanti ai governi nazionali la sua linea politica e riuscire eventualmente a persuaderli ad intraprendere azioni politiche conseguenti. Ci si può riferire a questa forma di leadership condivisa dal Presidente della Commissione come alla agenda-setting leadership.

Le tre principali forme di leadership del Presidente della Commissione non sono prive di connessioni fra loro. L'esercizio della leadership mediatrice e di quella di definizione dell'agenda politica, ad esempio, può ben dipendere dalla precedente costruzione di una amministrazione efficiente, e quindi dalla leadership amministrativa. La leadership sull'agenda politica è spesso accompagnata dalla leadership mediatrice, perché senza costruire coalizioni e pervenire a compromessi è difficile promuovere una linea di intervento politico, e così via.

Quali sono allora le risorse e i vincoli che insistono sulla carica di Presidente della Commissione? È questa la prossima questione da trattare. I due aspetti saranno analizzati separatamente anche se presentano, evidentemente, strette interconnessioni.

\section{I vincoli al potere}

I Trattati di Roma e di Maastricht non definiscono nei dettagli il ruolo della Presidenza della Commissione. Essi non includono alcuna disposizione equivalente a quella del Trattato di Parigi, contenente il breve ma esplicito riferimento al ruolo del Presidente della Alta Autorità ${ }^{3}$. 
La mancanza di riferimenti alla Presidenza della Commissione riflette una mancanza di consenso sui poteri presidenziali fra i soggetti che hanno approvato i Trattati. Il potere del Presidente della Commissione fu dunque deliberatamente maldefinito rendendo così tale carica, da un lato, suscettibile di contestazione e, dall'altro, come la si è definita in precedenza, «malleabile».

La procedura attraverso cui il Presidente della Commissione si insedia nella sua carica mostra, comunque, il profilo relativamente basso che egli è in genere obbligato a tenere; l'articolo 161 del Trattato di Roma disponeva che il Presidente della Commissione dovesse essere nominato di comune accordo fra $\mathrm{i}$ governi degli stati membri per un periodo di due anni, che poteva essere rinnovato.

Si può notare anzitutto che la durata del mandato del Presidente della Commissione è ridotta a due anni dai sei previsti per il Presidente dell'Alta Autorità. Ciò è dovuto alla diffidenza dei governi dei sei originari partner comunitari. Alcuni di essi (in particolare gli stati del Benelux) temevano il ripetersi dell'esperienza della forte Presidenza di Jean Monnet presso l'Alta Autorità (Conrad 1989), mentre il governo francese, da parte sua, non era pronto ad appoggiare la creazione di un altro organismo sovranazionale con lo stesso potere dell'Alta Autorità, specialmente dopo il fallimento della Comunità Europea di Difesa (CED). E vero che, per consuetudine, il Presidente della Commissione (dopo Hallstein) è rimasto generalmente in carica per quattro anni, ma, da un punto di vista formale, il breve mandato statuito dal Trattato di Roma dava ai leader dei governi nazionali il potere di dimissionare il Presidente ogni due anni. Con ciò si stabiliva un forte controllo da parte dei governi nazionali sul Presidente della Commissione: se questi voleva vedersi rinnovare il mandato, era quasi obbligato ad essere sensibile ai loro umori.

Il Trattato di Maastricht fissa la durata in carica del Presidente della Commissione in cinque anni rinnovabili e non prevede che i governi degli stati membri possano dimettere il Presidente durante il suo mandato. A parità di altre condizioni, questa maggiore durata della permanenza in carica rende la po-

de l'administration des services et assure l'exécution des délibérations de la Haute Autorité» (art. 16). Jean Monnet sfruttò a fondo questa norma quando costruì l'Alta Autorità partendo da zero (Morgan 1992, 1). 
sizione del Presidente della Commissione più forte rispetto al passato.

La procedura di nomina del Presidente delineata nel Trattato di Roma non prevedeva alcun intervento del Parlamento Europeo. Fino alla ratifica del Trattato di Maastricht, dunque, il Parlamento non aveva alcun diritto costituzionalmente garantito di influenzare la scelta del vertice della Commissione, ma era stato in qualche misura coinvolto nella procedura di nomina in via di prassi. Al momento dell'inaugurazione della Commissione Thorn nel 1981, ad esempio, fu introdotta la pratica per cui la Commissione riceveva il voto di fiducia del Parlamento dopo aver sottoposto ad esso il suo programma di interventi politici. La Dichiarazione di Stoccarda del 1983 confermava questa prassi e, oltre a ciò, esortava gli stati membri a consultare il Parlamento sulla nomina del Presidente della Commissione, cosa che da allora è accaduta regolarmente. Le Commissioni Delors del 1985 e del 1989, inoltre, fecero il gesto simbolico di prestare il giuramento ufficiale presso la Corte di Giustizia solo dopo aver ricevuto il voto di fiducia del Parlamento Europeo (Jacobs et al. 1992, 226-227).

L'articolo 158 del Trattato di Maastricht ha introdotto una nuova procedura per la nomina del Presidente della Commissione. In un primo momento, i governi degli stati membri designano la persona che intendono nominare Presidente e prima di contattarla consultano il Parlamento. Poi, dopo aver consultato il designato alla Presidenza, nominano gli altri Commissari i quali, come un organo unico, insieme al Presidente, si sottopongono all'approvazione del Parlamento Europeo. In caso di voto positivo, i governi nominano il Presidente e distribuiscono di comune accordo i portafogli fra i Commissari. La scadenza del mandato della Commissione e del suo Presidente, inoltre, è stata «sincronizzata» con quella del Parlamento.

Questa procedura può essere considerata come un passo verso l'introduzione di un tasso superiore di responsabilità nel sistema di governo della Comunità Europea. Per insediarsi nella carica, il Presidente della Commissione ha adesso costituzionalmente bisogno dell'appoggio della maggioranza dei membri del Parlamento Europeo direttamente eletti dal popolo. Un ulteriore esperimento si è avuto allorché Delors, nel settembre 1994, ha accettato di rispondere alle domande liberamente poste in aula dai deputati europei. La Commissione Santer, poi, si è sottoposta ad una serie di interrogazioni parlamentari ed è stata 
indotta a cambiare in parte la distribuzione dei portafogli prima di prestare il giuramento ufficiale. Si è affermato che i membri del Parlamento Europeo hanno considerato questo procedimento come affine alla ratifica delle nomine governative da parte del Congresso degli Stati Uniti, e il Parlamento e la Commissione sono d'accordo nel considerarlo un modo di rinforzare la legittimità democratica di quest'ultima. Ad ogni modo, nel più ampio contesto delle relazioni legislativo-esecutivo, il Presidente della Commissione ha ancora limitazioni notevoli sia nelle sue risorse di potere che nella possibilità di rivendicare la sua legittimità democratica.

I partiti politici possono essere una risorsa potente per il leader di un governo democratico ${ }^{4}$. Qualsiasi esplicita comparazione della Comunità Europea con una democrazia compiuta è fortemente fuorviante, dato che la $\mathrm{CE}$ non può essere certo ritenuta tale. Ad ogni modo, al fine di evidenziare l'ampiezza del gap di risorse esistente tra il capo di un governo nazionale e il Presidente della Commissione, è necessario considerare le relazioni di entrambi con i partiti politici.

In una democrazia, il Presidente o il Primo Ministro possono contare sulla maggioranza dell'assemblea legislativa, grazie all'appoggio di un unico partito (come spesso nel Regno Unito) oppure di una coalizione di partiti (come in molti paesi europei occidentali). Anche il Primo Ministro di un governo di minoranza talvolta guida il più forte partito in parlamento (come si può osservare nella Danimarca dell'ultimo decennio); e un Primo Ministro con una base di potere debole in un governo di coalizione può avere il controllo di una frazione del partito più importante (è il caso di alcuni Presidenti del Consiglio democristiani in Italia). I Presidenti americano e francese si troverebbero in una posizione molto più debole di quella prevista dal disegno costituzionale dei loro paesi senza una maggioranza del loro partito che li sostenga al Congresso o nell'Assemblea $\mathrm{Na}$ zionale. Finanche il capo del Consiglio Federale svizzero - forse la figura di capo di governo democratico la cui posizione istituzionale più si avvicina a quella del Presidente della Commissione Europea - prova a tirare dalla sua parte i parlamentari del suo partito (Hughes 1962, 93). Il punto centrale qui è che, in

4 In uno studio comparato sui Primi Ministri dei Paesi dell'Europa occidentale, G.W. Jones ha concluso che «la risorsa politica più importante di tutte è il partito» (1991b, 164). 
una democrazia, il numero di parlamentari sui quali un Primo ministro può contare si riflette direttamente sulla sua influenza nel tradurre effettivamente le sue linee programmatiche in provvedimenti dotati di forza normativa.

$\mathrm{Al}$ Presidente della Commissione manca in maniera manifesta la risorsa di un partito politico, e anche se l'importanza di questo elemento non deve essere esagerata poiché, come si è visto, tale risorsa può far difetto anche a molti capi di governo democratici, va messo in evidenza il fatto che il Presidente della Commissione non guida alcun partito né frazione politica. Di conseguenza non è in grado di contare su alcuna forza parlamentare per sostenere le sue linee di intervento politico.

Dei tre Presidenti succedutisi dopo l'introduzione dell'elezione diretta del Parlamento Europeo, Delors sembra essere stato l'unico ad aver ricevuto dal Parlamento qualcosa di più che un frettoloso apprezzamento. Jenkins, che era originario di un paese con forti tradizioni parlamentari, inizialmente si aspettava molto dall'elezione diretta dei deputati, ma alla fine fu sostanzialmente deluso dal debole ruolo giocato dal Parlamento Europeo (Jenkins 1989, 90 e 193-194). Thorn era appassionato di questioni istituzionali e per questo motivo rispettato dai federalisti più accesi nel Parlamento Europeo (Burgess 1989, 139). Considerati la sua grigia performance e il suo retroterra politico di liberale, che gli poteva guadagnare l'appoggio di un gruppo minoritario nel Parlamento Europeo, non si può dire che godesse di un forte prestigio.

Al contrario dei suoi predecessori, Delors ha avuto relazioni generalmente buone con il grosso dei membri del Parlamento. In primo luogo, la sua passata esperienza politica nel Partito Socialista Francese e la sua fede cristiana hanno giovato alla sua stretta relazione con il Gruppo Socialista e con il Gruppo Popolare, la cui forza è pari al 60-65\% dei seggi del Parlamento Europeo. In secondo luogo, prima di assumere la carica di Ministro delle Finanze con Mitterrand nel 1981, Delors era stato egli stesso un parlamentare europeo eletto e godeva di alta considerazione come Presidente del Comitato del Parlamento Europeo per gli Affari Economici e Finanziari. Infine, Delors ha deliberatamente puntato a guadagnare il maggiore sostegno possibile da parte del Parlamento Europeo, in particolare al momento di varare nuove iniziative politiche che incontravano l'opposizione di alcuni governi, come nel caso della Carta So- 
ciales. Così, per quanto non paragonabile ai leader di governi parlamentari, la stretta associazione di Delors con i due partiti più grandi del Parlamento Europeo ha costituito per lui una risorsa preziosa. Va messo in evidenza che l'importanza di questa risorsa «partitica» è in crescita, in quanto anche il Parlamento Europeo ha acquisito nuovi poteri.

Difficilmente la Comunità può suscitare la passione popolare. Mancando di una lingua comune, o di mass media di portata continentale, non può sostituire gli stati membri, ognuno dei quali ha connessioni con la volontà e il sentimento popolare molto più solide di quelle che può avere la $\mathrm{CE}$. Si può persino dire che la democrazia negli stati membri della Comunità funzioni in modo da rafforzare la loro legittimità nazional-popolare. Ogni volta che un popolo vota per le elezioni politiche o per un referendum, esso si rende conto di quello che decide: una scelta nazionale su determinate questioni o per un leader del proprio paese, non di altri. Nel fare ciò, ogni popolo può ripetutamente avere il sentimento, anche se in maniera vaga, del limite della propria influenza, confinata entro le frontiere nazionali ${ }^{6}$.

Una possibile comparazione può essere fatta con la Svizzera, dove alcune fondamentali linee di frattura culturali, linguistiche o etniche, rendono difficile integrare la società (e quindi dove la struttura della leadership è deliberatamente dispersa). Ma persino in Svizzera, l'esistenza di referendum istituzionalizzati e condotti a livello nazionale permette al popolo di esprimere la propria opinione in maniera diretta ed, eventualmente, di decidere la posizione del proprio paese in merito a determinate questioni. La Comunità Europea come entità politica a sé manca invece dei canali di collegamento fra popolo ed élites, dato che il Parlamento Europeo direttamente eletto ha finora avuto solo una debole incidenza politica.

Commentando la situazione della democrazia in Europa, Jürgen Habermas ha recentemente sostenuto:

5 Hallstein fece la stessa cosa nel 1964, allorché varò la cosiddetta Initiative. In una maniera in qualche modo provocatoria, giunse addirittura a renderla nota prima al Parlamento che al Consiglio. In quel caso, peraltro, il sostegno del Parlamento (non ancora eletto direttamente) non aiutò molto il Presidente.

6 In connessione a questo argomento, si deve tenere presente che la cittadinanza europea introdotta dal Trattato di Maastricht permette agli individui di altri stati membri di partecipare, nel paese europeo diverso dal proprio in cui essi risiedono, solo alle elezioni locali ed europee, e non a quelle politiche nazionali. 
(...) gli stati nazionali pongono meno problemi per la loro pretesa ad una sovranità ultima che per il fatto di aver delimitato il processo democratico in modo pressoché esclusivo all'interno delle loro frontiere. In una parola, fino ad oggi lo spazio pubblico è rimasto confinato al livello degli stati nazionali (Habermas 1992, 31).

I movimenti e i processi democratici sono dunque fondamentalmente monopolizzati dagli stati nazionali. Lo spazio pubblico resta essenzialmente nazionale, e questo continua a rendere la Comunità fortemente frammentata lungo le linee delle nazionalità degli stati membri.

In questo contesto, il rafforzamento del controllo del Parlamento Europeo sul Presidente della Commissione non rende automatica l'instaurazione di una legittimità democratica. Le procedure devono essere «riempite» dall'azione concreta dei movimenti democratici, in un processo che va dal basso verso l'alto. Senza partecipazione popolare e senza un intervento, almeno in alcune occasioni, di movimenti civici, un sistema politico non può essere considerato democraticamente legittimo.

Ė improbabile che ciò accada nella Comunità Europea. Quale sintesi politica confederale o (al massimo) consociativa, non ha mai favorito la formazione di un sentimento europeo sufficiente a sostenere un intero sistema democratico, parlamentare o presidenziale, a livello continentale, né ha avuto l'intenzione di farlo. C'è certamente stato un cambiamento, lento e limitato, nel senso dell'inserimento delle masse nel processo di integrazione continentale, specialmente dal momento dell'introduzione dell'elezione diretta del Parlamento Europeo. Ma ciò è lontano dall'instaurare una legittimità democratica ${ }^{7}$. Per esempio, anche se Delors, come si è detto in precedenza, ha tratto sostegno dai gruppi socialista e popolare del Parlamento Europeo, questo non significa che egli fosse democraticamente legittimato quale Presidente della Commissione, poiché quegli stessi gruppi sono poco più che confederazioni di partiti nazionali con affiliazioni politiche simili. Date queste circostanze, appare dunque chiaro come il Presidente della Commissione operi senza stretti legami con la società europea, e come il raggio della sua attività resti fondamentalmente confinato al livello delle élites.

La lista dei vincoli imposti al Presidente della Commissione non si esaurisce qui. L'articolo 1 del Regolamento Interno ${ }^{8}$ del-

7 Per un punto di vista simile a quello qui sostenuto, vedi Weiler $(1992,19-20)$.

8 «Règlement intérieure de la Commission», Journal officiel des Communautés eu- 
la Commissione afferma il principio collegiale, riferendosi al Presidente come ad un primus inter pares. Questa è diventata una norma largamente accettata nella Commissione. In comparazione con i ministri nazionali nei loro gabinetti, è molto più probabile che i Commissari discutano e risolvano i problemi davanti al Collegio che in incontri bilaterali con il Presidente? Torneremo su questo aspetto in seguito, quando considereremo il modello generale di relazioni fra il Presidente e i Commissari.

Il Trattato di Roma prevedeva che gli altri Commissari dovessero essere nominati di comune accordo da parte dei governi degli stati membri, per una durata di quattro anni. La scelta dei Commissari era pertanto nelle mani degli stati membri, il che effettivamente lasciava il Presidente della Commissione privo di una risorsa potente, ovvero la possibilità di distribuire posti di potere. Così, per esempio, persino Jenkins, che guidò con relativo successo una campagna volta ad influenzare la scelta dei suoi colleghi, vide alcuni dei 12 Commissari nominati contro la sua volontà (Jenkins 1989,9 ). Oltre a ciò, al Presidente della Commissione manca anche il potere di dimissionare i membri del Collegio. Solo la Corte di Giustizia, su richiesta della Commissione o del Consiglio, può, in circostanze eccezionali, dichiarare decaduto un Commissario ${ }^{10}$.

Il Presidente della Commissione può dunque in teoria trovarsi circondato da colleghi impostigli dai governi degli stati membri, poco propensi ad assecondarlo se non ostili; e per giunta non può rimuoverli. Si tratta di un vincolo considerevole alla discrezionalità del Presidente.

Il Trattato di Maastricht ha previsto un maggiore coinvolgimento del Parlamento Europeo nella nomina dei Commissari, ma per quanto riguarda il Presidente ha semplicemente obbligato i governi degli stati membri a consultarlo in merito alla loro scelta. Questo obbligo non sembra finora aver fatto una grande differenza.

Il Presidente della Commissione ha una limitata influenza

ropéennes, n. 17, 31 janvier 1963. La Commissione Delors ha modificato il Regolamento Interno nell'aprile 1989 e nel febbraio 1993 e ha inoltre approvato delle modalités d'application del Regolamento nel maggio 1993. Vedi Commissione (1993a e 1993b).

9 Il governo finlandese è quello che relativamente a questo aspetto presenta le maggiori affinità con la Commissione. Vedi Blondel (1991).

10 Solo un Commissario, Albert Borschette, è stato dichiarato decaduto attraverso questa procedura. Era in coma e morì poco dopo il suo dimissionamento nel 1976. 
anche sulla distribuzione dei portafogli fra i Commissari. Ogni decisione in questo campo è formalmente una decisione collettiva presa dal Collegio dei Commissari, ma il Presidente, oltre al suo Capo di Gabinetto, è probabilmente la sola persona che conosce tutte le aspirazioni dei membri del Collegio e quelle dei loro governi nazionali, come anche la disponibilità dei posti. $\mathrm{Ci}$ si aspetta dunque che sia il Presidente a smussare ogni conflitto di vedute ed a formare un team compatto.

Per questo motivo, la Presidenza della Commissione diviene il luogo di pressioni dirette ed indirette da parte dei Commissari e dei loro governi, e si deve rilevare che la risorsa dell'influenza sulla distribuzione dei portafogli si è sovente dimostrata negativa. Ciò perché spesso il Presidente fallisce nel comporre le diverse domande, e pertanto la sua autorità e competenza come esperto chairman possono essere messe in dubbio. Inoltre, lo scontento di alcuni fra i Commissari può essere dannoso per la coesione del Collegio.

Il compito è diventato particolarmente difficile dopo il 1989: il numero dei Commissari è aumentato mentre il numero delle Direzioni Generali è rimasto più o meno lo stesso; inoltre la crescita dello status dei Commissari e dell'attenzione dei media nei loro confronti ha inflazionato la domanda per un portafoglio ${ }^{11}$. Tuttavia, seguendo l'esempio della prima Commissione Delors, il nuovo Presidente Jacques Santer - con il suo efficiente Capo di Gabinetto Jim Cloos - è riuscito a venire a capo della distribuzione dei portafogli prima del suo insediamento formale nella carica.

L'indisponibilità di una risorsa di patronage costituzionalmente garantita, e le possibili conseguenze negative della influenza del Presidente sulla distribuzione dei portafogli, peraltro, non devono essere esagerate. Spesso neanche i leader governativi possiedono queste risorse. Il Presidente svizzero eletto per un anno - è forse il caso estremo di scarsa influenza sul personale ministeriale e sulla distribuzione dei posti. In Italia e in Olanda il Primo Ministro non ha altra scelta che accettare per i ministeri chiave le nomine dei partner coalizionali. Persino il Cancelliere tedesco non ha mano libera nella scelta dei ministri. In tutti questi casi, il premier non può permettersi

11 Intervista con Pascal Lamy, Capo di Gabinetto di Delors, Bruxelles, 19 aprile 1994. 
un rimpasto senza mettere in pericolo la coalizione. La «debolezza» della Presidenza della Commissione, almeno da questo punto di vista, è dunque relativa.

Un altro aspetto della «debolezza» della Presidenza, da considerarsi alla luce di altre esperienze istituzionali, è l'eterogeneità e la frammentazione della struttura amministrativa della Commissione. Jean Monnet, nel creare l'Alta Autorità, aveva concepito ogni dipartimento come altamente specializzato nei suoi compiti (Morgan 1992, 3-5). Walter Hallstein, quale primo Presidente della Commissione della Comunità Economica Europea, ha istituito la divisione verticale dell'apparato, pur provando - peraltro senza successo - a garantire il coordinamento facendo gestire una stessa area di intervento a tre Commissari (Noël 1992, 147-150). Le competenze ufficiali delle Direzioni Generali (DG) - spesso in parte sovrapposte a quelle di un Commissario - hanno finito per delimitare dei domìni simili a «baronie quasi-feudali» (H. Wallace 1983, 59-60). La potente DG6 competente per l'agricoltura, ad esempio, è legata più strettamente con le controparti nazionali, con le industrie e i sindacati agricoli che con le altre Direzioni Generali. Una tale eterogeneità e frammentazione non facilita di certo il lavoro del Presidente, ma non è affatto peculiare alla CE ed è senza dubbio comparabile a quella delle amministrazioni italiana ed olandese.

Un ulteriore elemento di difficoltà per l'azione del Presidente della Commissione è la diversa estrazione socio-politica dei Commissari. A norma dell'art. 157 del Trattato di Roma (art. 10 del Trattato di Fusione), il Collegio deve includere almeno un Commissario per ogni stato membro. Nel 1994, ad esempio, del Collegio facevano parte un greco ortodosso, un cattolico portoghese e un protestante danese, le cui visioni del mondo presentavano rilevanti differenze. Inoltre, la composizione del Collegio è tale che le affiliazioni politiche dei suoi membri devono essere bilanciate con attenzione, in particolare fra popolari, liberalconservatori e socialisti (la consuetudine nei paesi più grandi è quella di vedere rappresentate nella Commissione sia le forze di governo che quelle di opposizione). La Commissione può così comprendere al tempo stesso un fermo assertore del libero mercato di matrice anglosassone e un sostenitore egualmente convinto dell'intervento statale proveniente da un paese di tradizioni giacobine. L'incremento del numero degli stati membri ha accresciuto, infine, il numero delle lingue 
ufficiali della Comunità e con esso la difficoltà di comunicazione fra i Commissari ${ }^{12}$.

Per questo insieme di ragioni la Commissione Europea può essere concepita come una sorta di «grande coalizione». In prospettiva comparata, la Svizzera può servire ancora una volta come il modello più prossimo, poiché il suo governo federale è composto da ministri con tradizioni diverse di lingua e di cultura, oltre che distinte appartenenze politiche (Blondel e Bardi 1991, 1 e 21-22), per quanto nell'esperienza del governo elvetico i problemi di comunicazione (linguistica, culturale, sociale o politica) non siano così acuti come nella Commissione.

Dati questi vincoli interni (e, come si vedrà tra breve, le risorse della carica), quale tipo di relazione si può osservare fra il Presidente della Commissione e i suoi colleghi Commissari? Ci si può riferire a tre modelli generali: 1 ) subordinazione; 2 ) conflittualità; 3) partnership ${ }^{13}$.

Il caso della subordinazione dei Commissari al Presidente sembra il meno attendibile. I Trattati di Roma e Maastricht, come si è detto, non conferiscono al Presidente della Commissione nessun diritto costituzionale specifico rispetto al Collegio dei Commissari (al contrario di quanto succedeva per la Presidenza dell'Alta Autorità). Il Regolamento Interno della Commissione, inoltre, ha come prima regola quella della collegialità, e pertanto si riferisce al ruolo del Presidente come a quello di un primus inter pares. Ne consegue che il Presidente della Commissione ha un solo voto nel Collegio, esattamente come gli altri Commissari, e può dunque trovarsi sconfitto in una votazione importante ad opera della maggioranza del Collegio, il che è occasionalmente successo. Il principio collegiale infine è largamente ed effettivamente sostenuto da parte degli stessi Commissari (Blondel e Bardi 1991).

Ciononostante, come si vedrà, il Presidente detiene alcune risorse attraverso cui esercitare una propria influenza sui Commissari. Ciò lascia spazio per uno scenario di subordinazione, soprattutto quando la personalità ed il prestigio del Presidente

12 Il Presidente Malfatti, che aveva qualche difficoltà col francese, si trovò ad essere in qualche modo isolato dai suoi colleghi (come anche ad avere poca familiarità con l'ambiente politico di Bruxelles), il che costituì una delle molte ragioni per cui decise di non completare il suo mandato presidenziale e di tornare alla politica italiana. Materiale tratto da interviste.

13 Mi rifaccio ad uno schema formulato da Vincent Wright (1989) nella sua analisi delle relazioni fra Presidente della Repubblica e Primo Ministro in Francia. 
appaiono irresistibilmente forti agli occhi di certi Commissari. Prendiamo l'esempio del rapporto fra Delors e Karel van Miert. Van Miert - uno dei giovani e dinamici Commissari nella seconda e terza Commissione Delors - ambiva a presentarsi come un protégé del Presidente. Per poterlo apparire, van Miert concesse al Presidente molte delle priorità di intervento del suo portafoglio della concorrenza e, per molto tempo, sostenne il Presidente nelle discussioni nel Collegio. Dunque il rapporto fra Delors e van Miert può essere visto come un rapporto di subordinazione del secondo al primo. E altrettanto può dirsi del rapporto fra Delors ed Henning Christophersen, suo vicepresidente.

Una relazione conflittuale fra il Presidente e i Commissari ha tre possibili fonti: 1) linee di intervento politico; 2) competenze; 3) personalità. Un esempio calzante della prima fonte di conflitto è la disputa fra Hallstein e Robert Marjolin sull'Initiative del 1964. Marjolin era convinto della rischiosità della proposta del Presidente, ma alla fine lasciò campo libero ad Hallstein, il quale si assicurò il sostegno della maggioranza del Collegio (Marjolin 1989; Newhouse 1968). I conflitti di competenza possono insorgere specialmente quando il Presidente interviene in affari di competenza del portafoglio di altri Commissari. Jenkins, per esempio, ebbe grandi difficoltà con Ortoli, il suo vicepresidente incaricato degli affari monetari, allorché varò un'iniziativa per l'integrazione monetaria senza averlo consultato (Jenkins 1989, 173 e ss.). Anche il risentimento che Delors creò in Frans Andriessen e Ray MacSharry derivò in parte dai suoi interventi sui negoziati del GATT, che ricadevano formalmente nella competenza dei due Commissari. Infine, benché sia difficile districare il fattore della personalità dalle altre fonti di conflittualità, va sottolineato che l'alchimia personale gioca un proprio ruolo. Quando il Commissario Ralf Dahrendorf, sotto lo pseudonimo «Wieland Europa», criticò pubblicamente il Presidente Malfatti, non intendeva probabilmente portare un attacco personale, ma basava comunque le sue osservazioni su una valutazione negativa del Presidente ${ }^{14}$. Il rapporto personale fra Delors e Carlo Ripa di Meana non è mai stato facile, già prima che uno scontro sulla politica ambientale provocasse le dimissioni del secondo (Grant 1994, cap. VI e VIII). 
La categoria della partnership indica invece una relazione che determina un rafforzamento reciproco fra il Presidente e i Commissari. La si può osservare nella maggior parte del periodo della Presidenza Hallstein. Alcuni Presidenti hanno formato una sorta di «circolo interno» con alcuni fra i Commissari, stabilendo con loro un'alleanza. Jenkins, per esempio, si trovò in particolare sintonia con Etienne Davignon, Finn-Olav Gundelach e Lorenzo Natali (Jenkins 1989, xiii e ss.). E anche Delors, nella sua prima Commissione, strinse stretti rapporti con Natali, Lord Cockfield e (all'inizio) con Andriessen (Ludlow 1991, 119). Soprattutto la relazione Delors-Cockfield può essere vista come un buon esempio di partnership: senza comunità linguistica (entrambi parlavano poco la lingua dell'altro), ma con reciproco rispetto, i due (e i loro Gabinetti) formarono una solida alleanza per varare il programma del Mercato Interno, pur se non mancarono occasioni di disaccordo sull'implementazione del programma e sulla politica sociale ${ }^{15}$.

Un ultimo elemento da considerare è la Corte Europea di Giustizia. Si tratta, di solito, di un alleato della Commissione nel promuovere l'integrazione europea. Tuttavia, si può anche guardare ad essa come ad un potenziale vincolo per la Presidenza della Commissione.

Di solito la Commissione e il suo Presidente sono attenti, nell'intraprendere delle iniziative politiche, ad osservare la legislazione comunitaria. A tale scopo ogni proposta, dalla sua prima formulazione in poi, è esaminata dal Servizio Giuridico interno (che è sotto il controllo presidenziale, come si vedrà tra breve). Può avvenire, ad esempio quando il Presidente sostiene fortemente una proposta o quando c'è una particolare urgenza, che il parere del Servizio Giuridico risulti in qualche modo «conciliante». In eventualità del genere, si ha allora la possibilità che la Corte blocchi l'iniziativa, alla luce della sua non conformità al sistema normativo della Comunità. Questo è successo, per esempio, quando i giudici della Corte hanno messo in questione la legalità dello Spazio Economico Europeo, la cui Corte unificata con l'integrazione dei rappresentanti degli stati dell'EFTA poteva inficiare la supremazia della Corte Europea di Giustizia. Come è ampiamente noto, lo Spazio Economico Europeo (SEE) era una creatura di Delors e Lamy, che l'aveva- 
no varato dopo una discussione con il premier norvegese Gro Harlem Brundtland, senza consultare nella maniera appropriata il Collegio.

Le risorse interne di potere

Pur con questi vincoli al suo potere, il Presidente della Commissione detiene comunque delle risorse attraverso cui esercitare la sua leadership all'interno o all'esterno. In questa sezione si analizzano le risorse del Presidente nei confronti dei suoi Commissari attraverso un esame del Regolamento Interno della Commissione e con l'aiuto di alcuni esempi storici. Nella sezione seguente si prenderanno in esame le risorse esterne del Presidente della Commissione.

L'art. 3 del Regolamento Interno della Commissione specifica che il Presidente convoca e presiede le sedute del Collegio (formalmente chiamato «la Commissione»). Questo ruolo è rafforzato dall'art. 4, in cui si stabilisce che il Presidente predispone l'ordine del giorno delle sedute. Lo stesso articolo precisa che se un Commissario intende porre un nuovo argomento all'ordine del giorno dovrà informarne il Presidente con nove giorni di preavviso e che nei casi urgenti in cui questo termine non può essere rispettato la decisione ultima spetta al solo Presidente. Il Presidente può inoltre modificare durante le sedute le proposte avanzate dai membri della Commissione, mentre i membri hanno la possibilità di fare altrettanto solo nelle aree di competenza dei loro portafogli.

Nella sua qualità di chairman delle sedute del Collegio, pertanto, il Presidente della Commissione si trova spesso nella possibilità di orientare le discussioni o, almeno, di esercitare una particolare influenza sulla loro procedura. Egli è la sola persona (oltre al suo Capo di Gabinetto) che di solito vede tutti i documenti di lavoro in anticipo. Può intervenire in ogni momento dal banco della Presidenza. Decide chi deve prendere la parola $\mathrm{e}$ in quale ordine. Ha inoltre la possibilità di ricapitolare le discussioni. Quando sono usati in maniera estensiva, questi poteri aiutano il Presidente a dirigere le decisioni collettive nella direzione desiderata.

Un Presidente forte è poi in grado di influenzare il contenuto dei verbali. L'articolo 9 del Regolamento Interno statuisce che le bozze dei verbali approvate dal Collegio devono essere 
autenticate dal Presidente (e controfirmate dal Segretario Generale). Ciò non gli dà alcuna autorizzazione a cambiare il contenuto dei verbali, ma Delors, e in particolare il suo Capo di Gabinetto Lamy, sono stati frequentemente in grado di manipolare i verbali a loro favore, intervenendo nel processo di drafting degli stessi da parte del Segretario Generale (Grant 1994, 103).

Il Presidente ha formalmente il diritto di controllare il Segretariato Generale attraverso il suo vertice, il Segretario Generale. L'art. 15 del Regolamento Interno della Commissione stabilisce che il Segretario Generale ha il dovere di assistere il Presidente.

L'ambito delle attività del Segretario Generale è esteso. Con la collaborazione di uno staff di circa 200 persone (di grado «A»), aiuta il Presidente a preparare le sedute del Collegio, prestando particolare attenzione agli aspetti formali; fa circolare $i$ documenti interni; cura le pubblicazioni ufficiali; firma insieme al Presidente i verbali delle sedute del Collegio; coordina le diverse attività delle Direzioni Generali e intrattiene contatti e negoziati con le altre istituzioni della Comunità (Coombes 1970, 249-251).

Questi compiti non sono di natura meramente burocratica. Il Segretario Generale si assicura infatti almeno due tipi di risorse di natura politica presiedendo le sedute dei Capi di Gabinetto e quelle con i Direttori Generali. Attraverso queste sedute, egli ha anzitutto il compito di coordinare i diversi punti di vista. Non si tratta di un compito facile, in quanto i Commissari e i Direttori Generali governano tradizionalmente su baronie quasi-feudali e sono quindi diffidenti verso qualsiasi coordinamento. Se il Segretario Generale riesce a svolgere bene il suo compito può però trasformare il Segretariato in un punto di riferimento obbligato dell'apparato della Commissione e accrescere così la rilevanza della sua posizione. In secondo luogo, il Segretario Generale presiede le sedute dei funzionari di più alto livello e ha così l'opportunità di alimentare la propria rete di contatti interni.

Non va infine dimenticato che il Segretario Generale e il suo vice hanno funzioni esterne, ad esempio come capi negoziatori con il COREPER, il Comitato dei Rappresentanti Permanenti degli stati membri presso la Comunità. Normalmente questo compito è assolto dal vicesegretario, ma quando il COREPER tratta questioni squisitamente politiche - ad esempio la revisione dei Trattati - è il Segretario Generale a partecipare 
alle sedute ${ }^{16}$. Il Segretario Generale ed il suo vice non solo trasmettono al COREPER le comunicazioni, le proposizioni e le decisioni della Commissione, ma le difendono, se necessario, vigorosamente. Poiché le deliberazioni del COREPER possono ben passare attraverso il vaglio del Consiglio senza grandi modifiche, si comprende l'importanza cruciale del loro lavoro per la Commissione.

Quindi, in teoria, il Presidente può esercitare una considerevole influenza politica, all'interno ed all'esterno della Commissione, se ha un capace Segretario Generale sotto il suo controllo. Nei fatti la situazione è spesso più complicata, e il rapporto fra Presidente e Segretario Generale non sempre ha giovato al potere presidenziale.

Anche nelle relazioni fra il Presidente della Commissione ed il Segretario Generale si possono concepire e osservare rapporti di subordinazione, conflittualità e partnership.

La subordinazione del Segretario Generale al Presidente della Commissione è in un certo senso quanto ci si aspetterebbe dalla lettera del Regolamento Interno. La realtà tuttavia è un po' più sfumata. Bisogna infatti tenere presente che il Presidente della Commissione non ha il potere di nominare e dimissionare il Segretario Generale e, quindi, che il Segretario Generale non è controllato dal Presidente.

Le relazioni tra un Presidente potente ed un Segretario Generale influente possono poi attraversare fasi di conflittualità. Tuttavia, non c'è alcuna possibilità che il Segretario Generale sostituisca il Presidente o abbia l'ambizione di farlo, poiché non è una fonte di alternative politiche coerenti a quelle del Presidente.

Il terzo modello di relazioni, la partnership, può essere considerato il più naturale. Il Presidente e il Segretario Generale possono cooperare, con effetti positivi sulla influenza di entrambi. Il Presidente ha un dovere pubblico, all'interno e all'esterno della Commissione, che può essere svolto meglio se il Segretario Generale lo sostiene, esercitando un'influenza discreta, in cooperazione con il Gabinetto presidenziale, nei rapporti bilaterali con i Commissari e i Direttori Generali. In altri termini, per il Presidente la cooperazione del Segretario Generale è assai preziosa.

16 Noël partecipò alle riunioni del COREPER per guidare le discussioni sul Trattato di Fusione e sull'Atto Unico Europeo; Williamson al negoziato per il Trattato di Maastricht. 
La Commissione Europea ha avuto solo due Segretari Generali. Emile Noël, francese, ha occupato la carica per circa trent'anni, dalla nascita della CEE (quando la denominazione della carica era quella di Segretario Esecutivo, prima dell'unificazione dei tre «esecutivi»). Noël ha esercitato una grande influenza, grazie alla lunga permanenza in carica e ai suoi molti contatti, per tutti gli anni Settanta e nei primi anni Ottanta (durante la debole presidenza Thorn). Con l'avvento di Delors alla Presidenza della Commissione, Noël si è trovato a competere con il Capo di Gabinetto di Delors, Pascal Lamy, fortemente appoggiato dallo stesso Presidente. Ne derivò un periodo di relazioni «conflittuali» tra Presidente e Segretario Generale, anche se Noël riuscì abbastanza presto a ritagliarsi un proprio specifico spazio di influenza, soprattutto nella negoziazione dell'Atto Unico Europeo con le controparti del COREPER. In tal modo, Noël costruì in breve tempo un rapporto di «partnership» con il Presidente.

Il secondo Segretario Generale (in carica dal 1987 a tutt'oggi) è David Williamson, un efficiente funzionario britannico, che ha avuto alti incarichi nei governi Thatcher e aveva già lavorato nell'amministrazione della Comunità ricoprendo la carica di vicedirettore generale presso la DG6. In una prima fase Lamy esercitò un certo controllo sul nuovo Segretario Generale, anche perché all'insediamento di Williamson si era da poco affermata la prassi secondo cui il Segretario Generale riferiva al Presidente attraverso il Capo di Gabinetto di quest'ultimo. Al termine della presidenza Delors, quando Williamson era in carica ormai da circa sette anni, il prestigio e il potere del Segretario erano fuori discussione. Dopo un periodo di (parziale) «subordinazione» il rapporto tra Williamson e il Presidente è andato evolvendo verso il modello della «partnership».

Se la risorsa rappresentata dal Segretario Generale non sempre è all'altezza delle aspettative del Presidente, quest'ultimo può contare su una risorsa più stabile e potente: il suo Gabinetto e, in particolare, il Capo dello stesso (Krenzler 1974; Donnelly e Ritchie 1994).

Come gli altri Commissari, anche il Presidente può costituire un Gabinetto o un ufficio di consiglieri personali incaricato di assisterlo, come è specificato nell'art. 14 del Regolamento Interno. Il sistema dei Gabinetti (cabinets) è stato modellato su quello della burocrazia francese e messo in pratica già nell'Alta 
Autorità CECA. Nella Commissione della CEE-CE, i Gabinetti sono diventati sempre più influenti.

Nei primi anni di vita della Commissione CEE, ogni Commissario aveva un Gabinetto composto da due membri. Temendo la degradazione del carattere collegiale e sovranazionale della Commissione (i membri del Gabinetto sono normalmente della stessa nazionalità del loro Commissario), Hallstein volle limitarne il numero, pur avvalendosi egli stesso, poco dopo, di due membri aggiuntivi a titolo di conseilleurs (Coombes 1970, 255). Da allora il Presidente ha un Gabinetto più esteso di quello degli altri Commissari. Una decisione del Collegio del 10 gennaio 1973 ha fissato a cinque il numero dei membri (di grado «A») di ogni Gabinetto, assegnandone al Presidente uno in più. Questa regola tuttavia non è stata rispettata: sia i membri che le funzioni dei Gabinetti sono aumentati in una misura tale che nel 1979 il Rapporto Spierenburg ha dato l'allarme contro l'eccessivo potere da essi assunto.

Ciò nonostante la «gabinettocrazia» è sopravvissuta in forma robusta. Al suo Gabinetto Jenkins aggiunse il Central Advisory Group, un piccolo organo di quattro consiglieri consultato sulle linee politiche. Il Gabinetto di Thorn comprendeva un settimo membro, mentre i membri del Gabinetto di Delors furono addirittura undici (contro i sette dei Gabinetti dei Commissari) ${ }^{17}$.

I membri di un Gabinetto sono «occhi ed orecchie» del loro Commissario: lo tengono informato sugli sviluppi giornalieri; lo avvertono quando qualche problema bolle in pentola; dividendosi i compiti, preparano documenti, argomenti e strategie che mettono il Commissario in condizione di assolvere le sue responsabilità quale membro del Collegio. Il Gabinetto, inoltre, tiene d'occhio da vicino le nomine del personale, in cui spesso si inserisce appoggiando i candidati del proprio paese. Stabilire un contatto del Commissario con il proprio paese di origine e con le altre istituzioni comunitarie (in particolare il Parlamento) è una parte egualmente importante del dovere del Gabinetto. I membri del Gabinetto, e il suo Capo in particolare, spesso sostituiscono il Commissario quando questi è nell'im-

17 Su iniziativa di Delors fu fissata nel dicembre 1984 una regola secondo cui ogni Gabinetto doveva includere un membro di nazionalità diversa da quella del Commissario. Se un Commissario rispettava questa regola avrebbe potuto inserire un settimo membro nel suo Gabinetto. 
possibilità di partecipare ad una seduta. In breve, la performance dei Commissari (Presidente incluso) dipende in misura notevole da quella del loro Gabinetto.

Nelle riunioni ordinarie settimanali dei Capi di Gabinetto (ogni lunedì, sotto la presidenza del Segretario Generale) si combattono tutte le battaglie interne più rilevanti, le cui conclusioni talvolta passano senza grandi modifiche alle sedute del mercoledì del Collegio. Molti problemi tecnici sono già risolti nelle riunioni speciali dei Capi di Gabinetto (Special Chefs).

Il Gabinetto presidenziale ha poi alcune responsabilità aggiuntive. Al contrario dei Gabinetti dei Commissari, il Gabinetto del Presidente copre fondamentalmente tutte le attività delle Direzioni Generali e segue da vicino l'attività dei portafogli dei Commissari. Da ricordare inoltre che le riunioni speciali dei Capi di Gabinetto sono di solito presiedute da membri del Gabinetto presidenziale, $\mathrm{i}$ quali hanno così l'opportunità di controllare ordine del giorno e procedure di tali sedute.

Il Capo di Gabinetto del Presidente è di fatto il suo vice. All'interno, egli dirige il Gabinetto presidenziale; sostenuto dal prestigio del Presidente, è una figura centrale nelle riunioni dei Capi di Gabinetto e lo diventa ancor più se riesce ad allearsi con il Segretario Generale, il quale presiede quelle riunioni; attraverso i membri del Gabinetto da lui guidato, può intervenire nelle riunioni speciali dei Capi di Gabinetto. All'esterno, il Capo di Gabinetto del Presidente è il principale legame fra il Presidente e il mondo che lo circonda: è in contatto con le figure chiave dei governi degli stati membri o con i loro consiglieri, specialmente quando si devono trattare questioni delicate.

Con questi speciali canali e risorse, e anche attraverso la maggiore dotazione del suo staff, il Gabinetto presidenziale e il suo Capo cercano di fare di tutto per realizzare all'interno della Commissione gli obiettivi del Presidente. I giorni (e le notti) lavorativi non sono limitati a quelli feriali e il carico di lavoro è tale che nel 1986 un consigliere del Presidente è morto di superlavoro. I membri del Gabinetto coltivano diverse reti di contatti interne al fine di influenzare le decisioni ad ogni livello: quando ricevono un input dal Presidente o dal Capo di Gabinetto, lo trasmettono a loro volta ai funzionari dei gradi inferiori che, quando cooperano con il Gabinetto presidenziale, possono aspettarsi ogni sorta di favore possibile, promozioni incluse. Per realizzare i desideri del Presidente, i membri del suo Gabinetto utilizzano qualsiasi mezzo, inclusa la fame, la fatica o 
l'esasperazione. Un membro del Gabinetto di Delors, per esempio, era famoso per l'abitudine di convocare le sedute più difficili il venerdì pomeriggio, in modo da rendere più «ragionevoli» i membri degli altri Gabinetti ansiosi di partire per il fine settimana (Ross 1993, 23).

Tutti i Presidenti della Commissione della CE hanno tenuto per sé il portafoglio del Servizio Giuridico ${ }^{18}$. Come il Segretariato Generale, questo servizio è «orizzontale» poiché ha il diritto di esercitare la supervisione su tutto l'ambito delle attività della Commissione. Qualsiasi iniziativa politica da parte dei Commissari o dei Direttori Generali è soggetta all'esame formale da parte del Servizio Giuridico. Attraverso il Servizio Giuridico il Presidente e il suo Gabinetto possono influenzare il processo decisionale interno. Come ha riportato un osservatore interno, «la collaborazione fra il Gabinetto del Presidente e i funzionari superiori del Servizio Giuridico può portare ad una notevole vitalità della funzione della formazione dell'agenda politica. Un parere favorevole del Servizio Giuridico su di un certo testo è un chiaro vantaggio politico» (Spence 1994, 107). Pertanto, il controllo sul Servizio Giuridico può essere considerato un'importante risorsa per il Presidente.

Un Presidente smaliziato ed il suo staff possono influenzare il parere del Servizio Giuridico per scopi politici ${ }^{19}$, anche se manipolare il parere e la tempestività dell'intervento del Servizio Giuridico è comunque pericoloso, poiché così facendo il Presidente si espone ai rischi di eventuali rivelazioni su queste indebite pressioni (Grant 1994, 101).

Il Servizio del Portavoce è un'altra sezione importante, cui è demandata la responsabilità delle pubbliche relazioni della Commissione. Tale servizio è sempre ricaduto tra i portafogli assunti direttamente dal Presidente, almeno dal momento dell'unificazione dei tre «esecutivi» nel 1967. Il capo del servizio ha un contatto quotidiano con il Presidente e accesso diretto alle sedute del Collegio.

Il grado del controllo presidenziale sulle pubbliche relazioni varia da un Presidente all'altro, o da un periodo all'altro nel corso della stessa presidenza. Circondato da molti Commissari

18 Il Presidente Hallstein non lo fece poiché all'epoca i tre «esecutivi» avevano un servizio giuridico comune fornito dall'Alta Autorità.

19 Materiale tratto da interviste. 
«baroni», Thorn non fu in grado di dirigere le pubbliche relazioni, né di parlare a nome dell'intera Commissione. Determinato a non ripetere la caotica gestione delle pubbliche relazioni della presidenza Thorn, Delors tentò di rafforzare il controllo presidenziale in questo ambito istituendo un singolo Portavoce Capo sotto la sua diretta supervisione, in modo che il Presidente potesse parlare a nome di tutta la Commissione. Nel primo incontro con i suoi futuri colleghi Commissari (Royaumon, dicembre 1984) Delors chiese esplicitamente ai futuri Commissari di non assegnare ad un membro del loro Gabinetto il ruolo di proprio portavoce. Anche se questa raccomandazione non venne seguita dai Commissari, Delors manifestava con essa l'intenzione di controllare le pubbliche relazioni. Nel 1990 Delors nominò Portavoce Capo Bruno Dethomas, un ex giornalista di «Le Monde», amico di vecchia data e fino a quel momento portavoce personale dello stesso Delors nelle sue relazioni interne: Delors sperava in tal modo di controllare più strettamente le pubbliche relazioni della Commissione. Questa mossa si dimostrò però controproducente poiché Dethomas era visto come un uomo del Presidente, tanto che non era in grado di parlare con l'autorità dell'intera Commissione, ma solamente per Delors stesso ${ }^{20}$.

Un ultimo organo da considerare è la Cellule de Perspective creata dallo stesso Delors nel 1989 sulle fondamenta del Central Advisory Group costituito durante la presidenza Jenkins. Mentre quest'ultimo organo disponeva soltanto di quattro consiglieri, la Cellule conta circa quindici membri e ha un proprio Direttore Generale. Suo compito fondamentale è fornire consulenza, formalmente alla Commissione, ma in effetti al Presidente, sulle politiche a medio e lungo termine. È ragionevole ritenere che, una volta raggiunto il limite di espansione del Gabinetto presidenziale, questi organismi siano stati creati per rafforzare il potere del Presidente in una forma diversa. Per questa ragione entrambi gli organi sono stati guardati con sospetto dalle altre Direzioni Generali.

E egualmente vero, però, che essi rispondevano anche ad un bisogno reale: nel momento in cui la Comunità si avventurava in nuovi settori, che il Trattato di Roma non aveva delineato chiaramente, c'era necessità di una «squadra» che fornisse al

20 Devo questa indicazione al prof. Paemen, Portavoce Capo della Commissione Delors fra il 1985 e il 1987. 
Presidente consigli sulle politiche a medio e lungo termine. In questo senso, la creazione del Central Advisory Group alla fine degli anni Settanta e quella della Cellule de Perspective alla fine degli anni Ottanta possono essere considerate come altrettante risposte alla necessità di ridefinire le priorità di intervento politico della Comunità in questi nuovi àmbiti. In tal senso, lo scopo centrale di queste unità era quello di rafforzare la capacità del Presidente nel formare l'agenda politica della Comunità.

I Presidenti hanno normalmente assunto il diretto controllo anche di alcuni portafogli. I primi Presidenti (Hallstein e Rey) erano responsabili dell'Amministrazione. Ciò corrispondeva, oltre che al precedente fissato dalla Presidenza dell'Alta Autorità, alla necessità di esercitare la leadership amministrativa durante gli anni di formazione dell'apparato burocratico. Hallstein, inoltre, ebbe l'ultima parola sulle nomine del personale nei ruoli di funzionario durante tutto il corso della sua presidenza, mentre Rey delegò questo compito ad uno dei suoi vice, fornendo un esempio che sarebbe stato seguito dai suoi successori (Coombes 1970, 160).

Lasciando da parte l'ufficio della Sicurezza (una carica marginale riguardante le guardie di sicurezza interne), il Segretariato Generale, il Servizio Giuridico e il Gruppo dei Portavoce hanno costituito una «riserva» presidenziale fin dalla presidenza Rey (si veda la tabella 1). Jenkins è stato il primo ad assumere l'incarico di un portafoglio addizionale, l'informazione (all'epoca niente più di una task force). Come abbiamo appena visto, Jenkins ha anche creato e diretto il Central Advisory Group, rimasto da allora un portafoglio di pertinenza presidenziale. Thorn assunse fra i settori di sua competenza gli Affari Culturali. Particolarmente interessato alla riforma monetaria e dei fondi di coesione, Delors ne trasferì le relative competenze sotto il suo controllo. Oltre che negli affari monetari, Delors stabilì il suo controllo diretto sulla gestione dei fondi strutturali, e in particolare sulla questione dei Programmi Integrati Mediterranei e sui problemi connessi all'allargamento della Comunità alla penisola iberica (Smyth e Smyrl 1995). Questa espansione del domaine reservé presidenziale sembra continuare anche dopo Delors: Santer è riuscito infatti ad ottenere l'ultima parola, pur se in congiunzione con altri Commissari, in tre aree cruciali: l'unione monetaria, la politica estera e di sicurezza comune, e gli affari istituzionali, ovvero i temi centrali della prossima Conferenza Intergovernativa fissata per il 1996. 
TAB. 1. I Presidenti della Commissione europea e i loro portafogli (al momento dell'insediamento)

\begin{tabular}{|c|c|c|c|}
\hline Presidente & Nazionalità & Apparteneza politica & Portafogli (*) \\
\hline $\begin{array}{l}\text { W. Hallstein } \\
\text { (10//1958-5/VII/1967) }\end{array}$ & Germania & democratico-cristiano & $\begin{array}{l}\text { Segretariato, Amministrazione, } \\
\text { Personale }\end{array}$ \\
\hline $\begin{array}{l}\text { J. Rey } \\
\text { (6/VII/1967-30/VI/1970) }\end{array}$ & Belgio & liberale & $\begin{array}{l}\text { Segretariato generale, Servizio giu- } \\
\text { ridico, Servizio del portavoce }\end{array}$ \\
\hline $\begin{array}{l}\text { F. M. Malfatti } \\
\text { (1/VII/1970-21/II/1972) }\end{array}$ & Italia & democratico-cristiano & $\begin{array}{l}\text { Segretariato generale, Servizio giu- } \\
\text { ridico, Servizio del portavoce, Si- } \\
\text { curezza }\end{array}$ \\
\hline $\begin{array}{l}\text { S. Mansholt } \\
(21 / 1 \amalg / 1972-5 / 1 / 1973)\end{array}$ & Olanda & laburista & $\begin{array}{l}\text { Segretariato generale, Servizio giu- } \\
\text { ridico, Servizio del portavoce, Si- } \\
\text { curezza }\end{array}$ \\
\hline $\begin{array}{l}\text { F. X. Ortoli } \\
\text { (6/L/1973-5/1/1977) }\end{array}$ & Francia & gollista & $\begin{array}{l}\text { Segretariato Generale, Servizio giu- } \\
\text { ridico, Servizio del portavoce, Si- } \\
\text { curezza }\end{array}$ \\
\hline $\begin{array}{l}\text { R. Jenkins } \\
(6 / \mathrm{I} / 1977-5 / \mathrm{I} / 1981)\end{array}$ & Gran Bretagna & laburista & $\begin{array}{l}\text { Segretariato generale, Servizio giu- } \\
\text { ridico, Servizio del portavoce, Si- } \\
\text { curezza, Informazione (in seguito } \\
\text { Central Advisory Group) }\end{array}$ \\
\hline $\begin{array}{l}\text { G. Thom } \\
(6 / 1 / 1981-5 / 1 / 1985)\end{array}$ & Lussemburgo & liberale & $\begin{array}{l}\text { Segretariato generale, Servizio giu- } \\
\text { ridico, Servizio del Portavoce, Si- } \\
\text { curezza, Cultura, Central Advisory } \\
\text { Group }\end{array}$ \\
\hline $\begin{array}{l}\text { J. Delors } \\
\text { (6//1985-23/I/1995) }\end{array}$ & Francia & socialista & $\begin{array}{l}\text { Segretariato generale, Servizio giu- } \\
\text { ridico, Servizio del portavoce, } \\
\text { Central Advisory Group (in seguito } \\
\text { Cellule de Prospective), Affari } \\
\text { monetari, Fondi strutturali, Servi- } \\
\text { zio comune «Interpretazione-Confe- } \\
\text { renze» }\end{array}$ \\
\hline $\begin{array}{l}\text { J. Santer } \\
\text { (dal 23/V/1995) }\end{array}$ & Lussemburgo & socialista & $\begin{array}{l}\text { Segretariato generale, Servizio giu- } \\
\text { ridico, Servizio del portavoce, Si- } \\
\text { curezza, Cellule de Prospective, } \\
\text { Affari monetari }{ }^{+} \text {, Politica estera e } \\
\text { di sicurezza comune }+ \text {, Affari istitu- } \\
\text { zionali (conferenza intergovernativa } \\
\text { 1996)+, Servizio comune «Interpre- } \\
\text { tazione-Conferenze» }\end{array}$ \\
\hline
\end{tabular}

+ In congiunzione con altri Commissari

Un Presidente la cui sfera di attività si espanda in maniera eccezionale può acquisire altri portafogli semplicemente subentrando in quelli formalmente attribuiti a Commissari la cui azione risulti inefficiente. Delors, tipicamente, tagliava fuori i Commissari che lavoravano meno trattando questioni specifiche direttamente o attraverso il suo Gabinetto. Gli esempi abbondano. A metà del 1985, Delors fece squadra con Noël, Ehler- 
mann, François Lamoureux e Riccardo Perissich, mettendosi in tal modo in condizione di partecipare in maniera effettiva al processo di redazione dell'Atto Unico. Ciò a dispetto dell'esistenza del Commissario responsabile per le questioni istituzionali (Carlo Ripa di Meana), il cui Capo di Gabinetto (Perissich) faceva parte del team di Delors. Nella seconda Commissione Delors, Patrick Venturini - il membro del Gabinetto di Delors responsabile per gli affari sociali - subentrò di fatto nel portafoglio della politica sociale all'inefficiente Commissario Papandoreou, e si disse ironicamente che era in effetti il suo vero Capo di Gabinetto ${ }^{21}$.

Ci sono molte reti di contatti informali all'interno della Commissione. Alcune sono organizzate su basi personali, altre hanno natura politico-ideologica. Un noto esempio del primo tipo è la rete di contatti personali che Noël aveva stabilito all'interno della Commissione. Un Presidente abile ha bisogno di utilizzare diverse reti di contatti personali al fine di realizzare $\mathrm{i}$ suoi scopi politici.

Un esempio rappresentativo di reti di contatti informali può essere ravvisato nel «Gruppo Istituzionale» formato nel 1984 per seguire le discussioni del Comitato Dooge sulle questioni istituzionali. Con Noël, Ehlermann, Perissich e Lamoureux come membri centrali, questo Gruppo venne poi formalizzato sotto la presidenza Delors, ponendosi come forza centrale della Commissione nel processo di formazione dell'Atto Unico ${ }^{22}$. Un altro esempio può trovarsi nei gruppi ad hoc sulla riunificazione tedesca che furono presieduti da Williamson e da Carlo Trojan, suo vice. Questi gruppi fecero molto per rendere l'accesso della Germania Est nella Comunità Europea fattibile e indolore, con ciò favorendo la riunificazione tedesca (Spence 1993). Delors si appoggiò di più sulla sua rete di contatti informali che sul Collegio.

Le risorse esterne di potere

Fra le risorse esterne del Presidente della Commissione, il Consiglio Europeo può essere la più potente di tutte. È vero che

21 Grant (1994) e materiale tratto da interviste.

22 Materiale tratto da interviste. 
il Presidente della Commissione deve la sua nomina ai membri del Consiglio Europeo. Una volta nominato detiene però i mezzi per mobilitare questo organo, e quindi i più alti e importanti decisori nazionali, al fine di far avanzare il processo di integrazione. Se, al contrario, non riesce a ottenere il sostegno del Consiglio Europeo per le sue politiche, il contributo della Commissione alla costruzione della Comunità avrà poche probabilità di essere significativo. Il successo o il fallimento della leadership del Presidente della Commissione dipende insomma largamente dalla sua performance presso il Consiglio Europeo.

Bisogna ricordare infatti che il Presidente della Commissione è membro non solo della Commissione, ma anche del Consiglio Europeo. Il Presidente della Commissione è presente agli incontri al vertice fra i leader dei governi europei fin dal summit di Roma del 1967. La Dichiarazione Solenne di Stoccarda del 1983 ha ribadito la pratica precedente, affermando che il Consiglio Europeo comprende i capi di stato e di governo e il Presidente della Commissione. L'Atto Unico ha poi confermato dal punto di vista formale l'esistenza del Consiglio Europeo, così come la piena membership in esso del Presidente della Commissione. Questa doppia appartenenza, da un lato, offre al Presidente della Commissione una base istituzionale da cui esercitare influenza a livello dei leader governativi e, dall'altro, lo aiuta a differenziarsi dagli altri Commissari e ad affermare la propria autorità nei loro confronti.

I primi Presidenti della Commissione erano in genere scettici, e talvolta persino ostili, nei confronti degli incontri al vertice fra i leader governativi. Hallstein affermò che «ogni azione parte dalla Commissione» $(1979,82)$. Era naturale per lui difendere le prerogative della Commissione, che comprensibilmente considerava minacciata dagli insistenti sforzi di De Gaulle volti a istituzionalizzare gli incontri di vertice europei. Quando gli fu permesso per la prima volta di partecipare al summit di Roma nel 1967, nel decimo anniversario del Trattato di Roma, egli difese con forza le istituzioni della Comunità ${ }^{23}$.

Con l'uscita di scena di De Gaulle, l'atteggiamento della Commissione nei confronti degli incontri al vertice cambiò. Durante la presidenza Rey, la Commissione «salutò con favore» ${ }^{24}$ il

23 Bollettino delle Comunità Europee (Bull EC), 4-1967, p. 5.

24 Bull EC, 1-1970, p. 16. 
summit dell'Aja del 1969. E nella fase preparatoria del summit di Parigi del 1972, la Commissione Malfatti approvò il summit «poiché solo con decisioni politiche prese al massimo livello la Comunità potrà fronteggiare i suoi compiti nella maniera più appropriata ${ }^{25}$. La Commissione fu poi in qualche misura coinvolta in occasione dei summit di Parigi (1972), di Copenhagen (1973), e ancora di Parigi (1974) (Werts 1992, 54 e 141). Dopo la sua istituzione nel 1975, anche Ortoli ebbe parole di apprezzamento verso il Consiglio Europeo (ivi, 142).

I contrasti continuarono, ad ogni modo, sulla questione della piena partecipazione del Presidente della Commissione ai summit. Per essere presente al summit dell'Aja del 1969, di importanza cruciale, Rey dovette lottare molto aspramente (Ivi, 141), mentre nel vertice di Parigi del 1972, Mansholt - Presidente ad interim dopo le dimissioni di Malfatti - non poté prendere parte alle sessioni come partecipante di pieno diritto $^{26}$. Ortoli ricevette un'accoglienza molto fredda al summit di Copenhagen del 1973, specialmente da parte del Presidente del Consiglio Europeo in carica, Anbker Jorgensen, che non gli permise di partecipare alle sedute in cui non si trattava di affari di specifica competenza della Comunità (ivi, 141-142). Come si vede, il Presidente della Commissione ebbe per un lungo periodo uno status di secondo piano nella successione degli incontri al vertice fra i leader europei.

Comunque, una volta che la consuetudine della partecipazione del Presidente della Commissione ai vertici intergovernativi si era andata consolidando, c'era solo bisogno di una personalità forte e capace perché la sua posizione si rafforzasse definitivamente. Jenkins fu il primo Presidente che capì interamente il potenziale del Consiglio Europeo. Dopo la sua prima partecipazione alle sue sedute, affermò:

Il Consiglio Europeo in sé è un organismo sorprendentemente soddisfacente, soprattutto perché in esso c'è un'atmosfera raccolta. C'erano solo venticinque persone nella stanza. (...) Ció rappresenta un notevole miglioramento rispetto alle pletoriche adunate che caratterizzano il Consiglio dei Ministri, in cui sono presenti fino a trecento persone in una vastissima sala, e dove si parla da un capo all'altro del tavolo come da un'estremità all'altra di un campo da football vuoto (Jenkins 1989, 74).

25 Bull EC, 8-1972, p. 15.

26 Le Monde, 20 ottobre 1972, p. 1. 
Proseguì osservando che il Consiglio Europeo offriva l'opportunità di «un incontro ristretto con piena pubblicità successiva, il che forse non costituisce una cattiva formula» (Ivi, 75). Jenkins, quindi, superò quasi completamente l'atteggiamento sospettoso dei suoi predecessori nei confronti degli incontri intergovernativi di vertice e cominciò invece a cercare un modo di trarre vantaggio da essi.

Quando poi varò la sua importante iniziativa per l'unione monetaria a Firenze nel $1979^{27}$, la maggioranza dei suoi Commissari e i membri dell'Ecofin (particolarmente i tedeschi), come anche i mass media, non reagirono in maniera entusiastica. Tuttavia, Jenkins si preoccupò poco del loro atteggiamento e continuò a premere sui capi di governo, in particolare sul Cancelliere tedesco Helmut Schmidt perché, secondo il suo punto di vista, sarebbero stati i leader di governo, e non i ministri dell'Ecofin, la stampa, o i Commissari, ad avere l'ultima parola. La sua iniziativa fu ripresa in seguito nei tre Consigli Europei del 1978 da parte del Cancelliere tedesco e del Presidente francese Valéry Giscard d'Estaing, e portò alla decisione di creare il Sistema Monetario Europeo (Ludlow 1982, cap. II).

Il punto da fermare è che nella sua azione Jenkins non solo mobilitò i membri chiave del Consiglio Europeo, ma superò anche la riluttanza e l'opposizione di Ortoli, vicepresidente della Commissione e responsabile per gli affari monetari (Jenkins 1989, parte I). Si delineava in forma embrionale un possibile impiego della doppia membership da parte del Presidente, allo scopo di inserirsi nel momento strategico della formazione dell'agenda politica della Comunità e di stabilire la sua autorità sugli altri Commissari.

Delors ha utilizzato in modo sistematico la doppia appartenenza, spostando il baricentro della Presidenza, secondo la propria convenienza, dalla membership della Commissione a quella del Consiglio Europeo. Delors ha attribuito maggiore importanza al Consiglio che alla Commissione, precisando in proposito:

La presenza a tempo pieno al Consiglio Europeo e il ruolo attivo che vi svolgo è per me un elemento importante per poter dirigere una Commissione che non è affatto facile da governare, dal momento che non sono che il pri-

$27 \mathrm{Al}$ contrario di quanto farà Delors, Jenkins avanzò la sua proposta per l'integrazione monetaria nell'Istituto Universitario Europeo e non all'interno del Consiglio Europeo. 
mus inter pares. Partecipare con pienezza di diritti al Consiglio Europeo mi conferisce infatti una certa autorità sui miei colleghi della Commissione (che ad essi piaccia o no). Ciò è stato uno shock per tutti coloro che condividevano le finalità della mia azione politica ma che avendo una visione «classica» delle strutture ritenevano che il Consiglio Europeo non fosse altro che una organizzazione intergovernamentale. Se io fossi stato un dogmatico, non sarei riuscito nel mio intento ${ }^{28}$.

Anche se Delors non è stato l'unico a riconoscere il potenziale del Consiglio Europeo, la sua osservazione rivela a qual punto egli lo abbia deliberatamente utilizzato al fine di stabilire la sua autorità nella Commissione. Il solo fatto di partecipare alle riunioni del Consiglio Europeo eleva infatti lo status del Presidente. Dopo aver ottenuto un mandato dal Consiglio, inoltre, il Presidente può dare maggior peso ai suoi interventi politici, che quindi possono difficilmente essere contestati dagli altri Commissari. In questo modo Delors ha stabilito una autorità senza precedenti della figura del Presidente all'interno della Commissione $e^{29}$.

Altrettanto importante è stato il successo di Delors nel rendersi indispensabile come membro del Consiglio Europeo: dalla seconda metà del 1985 è stata progressivamente istituita la prassi secondo cui ogni argomento veniva introdotto nella discussione davanti al Consiglio Europeo dal Presidente della Commissione. Per la semplice circostanza di agire al livello dei vertici governativi degli stati membri, Delors ha innalzato lo status del Presidente della Commissione quasi al rango di un capo di governo. Ne costituisce un esempio recente la sua iniziativa per l'adozione del Libro Bianco sulla crescita, la competitività e l'occupazione nel 1993: Delors non solo ha preso personalmente l'iniziativa in questo ambito, a dispetto dell'esistenza del Commissario responsabile per quelle materie, ma è riuscito anche a tagliare fuori il Consiglio dei Ministri Ecofin, rivolgendosi direttamente ai capi di governo (Hill 1993, 2).

La mobilitazione del Consiglio Europeo, infine, ha coinvolto il Presidente nella formazione dell'agenda politica della Co-

28 Intervista con Jacques Delors, Presidente della Commissione della Comunità Europea, Bruxelles, 30 giugno 1994.

29 Un ex vicepresidente che spesso ha assistito Delors al Consiglio Europeo ha osservato che molte volte Delors parlava «a titolo personale» su «argomenti che non erano ancora stati discussi nel Collegio». Intervista con il prof. Frans Andriessen, Bruxelles, 4 maggio 1994. 
munità. La maggior parte dei successi di Delors, come l'adozione del Pacchetto Delors I, l'istituzione del Comitato sull'Unione Monetaria Europea, e la formulazione e l'adozione del Libro Bianco del 1993, sono stati resi possibili attraverso l'utilizzo di questa risorsa. In conclusione, il Presidente della Commissione può realizzare molti dei suoi obiettivi politici attraverso la sua doppia membership, della Commissione e del Consiglio Europeo; e questa deve essere considerata come la sua maggiore risorsa esterna per esercitare la sua leadership (agenda-setting).

La Presidenza del Consiglio, il Segretariato del Consiglio e l'asse Parigi-Bonn sono invece risorse la cui natura può essere definita «a doppio taglio». Tutti e tre questi «attori» sono in una posizione tale da influenzare l'agenda politica della Comunità e, in particolare, le procedure del Consiglio Europeo. Un Presidente della Commissione che voglia mettersi in condizione di partecipare effettivamente alle funzioni di mediazione e di agenda-setting ha la necessità di contare sul loro sostegno. Se fallisce in ciò, la sua sarà probabilmente una presidenza debole.

Il Trattato di Parigi prevedeva una Presidenza del Consiglio tutto sommato modesta, da esercitare per tre mesi, a rotazione. Il Trattato di Roma ha esteso il periodo a sei mesi. Durante gli anni Sessanta e Settanta, la Presidenza del Consiglio ha assunto saldamente importanti funzioni di iniziativa politica, di mediazione nelle trattative, di punto di contatto con le altre istituzioni comunitarie e di rappresentanza della Comunità nelle relazioni esterne. Il crescente ruolo della Presidenza del Consiglio si deve in parte all'espansione degli affari del Consiglio, ma è dipeso anche dal vuoto scaturito dall'indebolimento della leadership della Commissione, soprattutto dopo la crisi del 19651966. L'importanza del ruolo della Presidenza del Consiglio è stata ulteriormente accresciuta dall'istituzionalizzazione del Consiglio Europeo nel 1975 e dall'applicazione ad esso della presidenza a rotazione (Wallace e Edwards 1976).

Tra le varie funzioni della Presidenza del Consiglio, la definizione dell'ordine del giorno e della presidenza delle sedute appare di particolare rilevanza. Se il Presidente della Commissione vuole affermarsi come uno degli attori principali della Comunità, non può quindi non tenere conto del ruolo giocato dalla Presidenza del Consiglio.

La misura in cui una Presidenza del Consiglio coinvolge la (Presidenza della) Commissione nella formazione dell'agenda politica della Comunità per sei mesi varia a seconda che sia de- 
tenuta dal governo di uno stato piccolo o di uno stato grande, di un paese pro-integrazione oppure di un paese anti-integrazione. In altri termini, il Presidente della Commissione ha più ampi margini d'intervento, ad esempio, con una presidenza belga, mentre un governo britannico tenderà a un esercizio esclusivo della direzione politica. Il Presidente della Commissione tenta di influenzare l'agenda politica nella direzione desiderata o attraverso alcuni canali prestabiliti, quali gli incontri semestrali con il governo che è sul punto di prendere la Presidenza del Consiglio, oppure attraverso contatti informali con il Presidente in carica.

Un buon esempio di cooperazione fra il Presidente in carica nel Consiglio Europeo e il Presidente della Commissione è fornito dal rapporto di collaborazione stabilitosi tra Delors e la presidenza belga nella seconda metà del 1993 allo scopo di dare nuovo impulso al processo di integrazione dopo la faticosa ratifica del Trattato di Maastricht. Attraverso un incontro nel giugno 1993, poco prima dell'insediamento della presidenza belga, il governo di quel paese e la Commissione discussero la via migliore per varare il Libro Bianco sull'integrazione e, su proposta di Delors, si decise che avrebbe dovuto essere il Consiglio Europeo e non l'Ecofin ad occuparsene. Ciò andava contro i desideri dei Ministri delle Finanze e, in particolare, dei ministri britannico e tedesco, oltre che a detrimento dello stesso Ministro delle Finanze del governo belga. I capi di governo approvarono il documento nel Consiglio Europeo di Bruxelles del dicembre 1993.

Il Segretariato del Consiglio aveva inizialmente un ruolo di secondo piano che non minacciava in nessun modo le prerogative della Commissione. Tuttavia, man mano che le funzioni ed il carico di lavoro della Presidenza del Consiglio sono andati aumentando, particolarmente dalla metà degli anni Sessanta, il Segretariato ha progressivamente acquisito una posizione più importante (Wallace e Edwards 1976, 536 e ss.; Nuallain 1985) e la sua incidenza (e in particolare quella del Segretario Generale) è ulteriormente cresciuta dall'inizio degli anni Ottanta. Ciò si può spiegare in base a tre fattori: legale-istituzionale, personale e politico.

In base ad una decisione del Consiglio ${ }^{30}$, il Segretario Gene-

30 Vedi la decisione del Consiglio del 26 settembre 1980, Official Journal of the European Communities (OJ), Vol. L, 261/16, 4 ottobre 1980. 
rale del Consiglio può anzitutto fornire al Presidente in carica delle speaking notes, che possono riguardare questioni di alto interesse politico all'ordine del giorno. Questo ovviamente conferisce al Segretario Generale una certa influenza. Un altro fattore istituzionale è derivato dall'allargamento della Comunità a stati più piccoli che, come la Grecia o, in misura minore, il Portogallo, sono stati fortemente dipendenti dal Segretariato per sbrigare gli affari del Consiglio durante i loro periodi di presidenza.

In secondo luogo, nell'ottobre 1980 Niels Ersbøll, ex rappresentante permanente danese, ha assunto il posto di Segretario Generale, che avrebbe poi lasciato nel 1994. La presenza di Ersbøll al Consiglio Europeo, la sua posizione, che lo metteva in grado di comprendere le sensibilità dei governi e di influenzare il Presidente in carica, così come la sua dettagliata conoscenza degli affari comunitari, lo hanno reso una figura affidabile per i governi degli stati membri (Howe 1994, 454). Tenendo presente tutto ciò, non è difficile capire il motivo della sua crescente influenza sugli affari comunitari. Nel 1985 egli ha lavorato a stretto contatto con la Presidenza della Commissione nel processo di redazione dell'Atto Unico; ha inoltre partecipato alla redazione di un documento di lavoro sull'Unione Politica per il summit del dicembre 1990 a Roma e, quando il Lussemburgo assunse la Presidenza nel 1990, Ersbøll e i suoi più stretti aiutanti (particolarmente il suo Capo di Gabinetto, P.S. Christoffersen, e il Direttore Generale del Servizio Giuridico del Consiglio, Jean-Claude Piris), in cooperazione con le massime autorità diplomatiche del paese (come Jim Cloos) e con il Rappresentante Permanente francese (Pierre de Boissieu), concretamente scrissero gran parte del Non-Paper del marzo 1991, che più tardi sarebbe diventato la base del Trattato di Maastricht (Cloos et al. 1993; Grant 1994). Così, il Segretario Generale si è affermato come una figura centrale nella Comunità.

C'è, infine, una ragione più strutturale alla base della crescita dell'influenza del Segretariato del Consiglio. La Commissione, sotto la presidenza Delors, perseguì abbastanza risolutamente l'espansione dei propri poteri e di quelli della Comunità. Tale tendenza fu simbolizzata nel 1988 da un'osservazione molto pubblicizzata di Delors secondo la quale nel 2000 circa 1'80\% della legislazione sociale sarebbe derivato dalla Comunità, affermazione che causò risposte negative non solo nel Regno Unito, ma anche in altri stati membri. Alcuni governi furono 
perplessi ed irritati per il modo in cui la Commissione acquisiva sempre maggiore visibilità come rappresentante esterna della Comunità sfruttando mandati che le venivano affidati relativamente a questioni interne, e la tendenza della Commissione a minimizzare o dissimulare tutto ciò peggiorò la situazione. Una serie di provvedimenti normativi relativi al mercato interno, poi, certamente aumentò la pressione legislativa sugli stati membri, rispetto ai quali questa legislazione si poneva come antagonista, anche se poi essa era ovviamente il risultato di accordi sottoscritti dagli stati stessi. Tutto ciò produsse, intorno al 1990, un sentimento di insoddisfazione, o persino ostilità, verso la Commissione. Gli stati membri, di conseguenza, cominciarono a guardare al Segretariato del Consiglio come ad un organo più affidabile in relazione ai loro bisogni ${ }^{31}$.

Dunque, il Segretariato del Consiglio può essere una risorsa per il Presidente della Commissione solo se e quando può diventare suo alleato. La crescita della sua influenza, comunque, può trasformare il Segretario in un «rivale», piuttosto che costituire una potenziale «risorsa». Di conseguenza, le possibilità del Presidente della Commissione di allearsi con lui, o di dominarlo, potrebbero diminuire. A questo scopo, il Presidente può sfruttare tuttavia la sua membership nel Consiglio Europeo, un'importante risorsa di cui il Segretario è privo. Oppure può mobilitare la tradizionale forza dell'asse franco-tedesco.

Fin dalla creazione della Comunità, l'asse franco-tedesco ha spesso indicato la rotta generale dell'integrazione europea ( $\mathrm{Si}$ monian 1985). Il succedersi dei governanti nei due paesi non ha incrinato seriamente la loro relazione. Questo è dovuto almeno in parte all'istituzionalizzazione delle relazioni fra $i$ due paesi che il Trattato dell'Eliseo del 1963 ha reso possibile. Il tandem Kohl-Mitterrand è così sopravvissuto senza il supporto di un'amicizia personale né di una lingua comune, che invece caratterizzavano il rapporto fra Schmidt e Giscard D'Estaing. La volontà dei leader dei due paesi di apparire come i veri ispiratori dell'agenda politica della Comunità si è rivelato sufficiente alla creazione di un fronte compatto.

Per la Presidenza della Commissione riuscire a mobilitare l'asse Parigi-Bonn è quanto mai rilevante. Tre attivi Presidenti

31 Materiale tratto da interviste. I sentimenti «anti-Commissione» cui si diede libero sfogo dopo il referendum danese del 1992 possono essere visti come una parte di questo trend strutturale. 
hanno avuto uno stretto rapporto con uno o entrambi i leader di Francia e Germania. Hallstein, per esempio, ha avuto un legame diretto con il Cancelliere Adenauer fino al 1963, anno in cui Adenauer lasciò la Cancelleria, anche se la drammatica caduta dello stesso Hallstein, inflittagli dal Presidente francese De Gaulle, esemplifica la natura «a doppio taglio» del ricorso all'asse Parigi-Bonn (Wallace 1993). Anche Jenkins ebbe un rapporto abbastanza buono con il Cancelliere tedesco Schmidt e, a dispetto della sua relazione non molto buona con il Presidente francese Giscard d'Estaing, poté contare sul sostegno di quest'ultimo nella sua importante iniziativa sull'unione monetaria (Jenkins 1989, 168-170).

La complessità della relazione di Delors con Mitterrand non impedì loro di intrattenere fruttuosi rapporti (Buchan 1991, 8; Milèsi 1985): alcune iniziative politiche come la Carta Sociale o il Media programme non sarebbero state concepibili se Delors non avesse potuto contare sul pieno sostegno del Presidente francese. Il Libro Bianco del 1993 fu approvato dal Consiglio Europeo di Bruxelles sulla base della strategia di Delors che il Presidente francese (così come la Presidenza dēl Consiglio) approvava caldamente. Il Cancelliere Federale Kohl, poi, propose Delors come Presidente della Commissione e la relazione amichevole fra i due, sulla base della quale Delors riuscì a far passare la sua linea sul bilancio comunitario nel 1988, si andò gradualmente rafforzando. Kohl, in qualità di Presidente del Consiglio in carica, sostenne anche l'idea di creare un Comitato sull'Unione Monetaria Europea presieduto da Delors. Il loro rapporto migliorò infine in modo decisivo allorché Delors, in anticipo praticamente su tutti gli altri politici europei (eccetto il premier spagnolo Felipe González), approvò senza condizioni l'unificazione delle due Germanie.

La posizione della Presidenza della Commissione può poi ricevere un forte sostegno dal prestigio acquisito dal Presidente nell'arena internazionale. Data l'ambiguità della carica, il prestigio internazionale può davvero costituire una risorsa importante per esercitare la leadership.

La contrastata presenza di Jenkins al summit del G7 fornisce un utile esempio dell'innalzamento del suo status e di quello della Presidenza. Jenkins, che spese molto tempo e molta energia durante i primi sei mesi del suo mandato per ottenere la partecipazione in quel meeting, affermò: 
L'unica questione dibattuta (...) era se io dovessi o no essere presente. L'assenza di Ortoli era stata ingiustamente vista come un colpo al suo prestigio e a quello della Commissione. Il ruolo che ci si aspettava che io avessi era in parte quello di ricostituire questo prestigio. Era davvero in gioco la mia credibilità come Presidente efficace (Ivi, 21).

Sostenuto dagli stati più piccoli, Jenkins si assicurò un posto, pur secondario con i Ministri delle Finanze, nel summit di Londra del 1977. Si trattò di un precedente prezioso per i suoi successori, in particolare per Delors che era egualmente desideroso di migliorare lo status della Presidenza.

Nel 1989 il prestigio di Delors ricevette nuovo alimento dal mandato, relativo al coordinamento degli aiuti verso i paesi dell'Europa Centrale ed Orientale, ricevuto dal summit del G7 di Arche. Si trattava non soltanto del riconoscimento della Comunità come importante attore internazionale, ma anche della conferma della sua già elevata reputazione dovuta al successo del Programma del 1992 del Mercato Interno.

Da quando la Comunità si è affermata come attore internazionale, i canali che il Presidente della Commissione può sfruttare per consolidare il suo status si sono moltiplicati. Il Presidente degli Stati Uniti George Bush, il Presidente del Consiglio Europeo Giulio Andreotti e il Presidente della Commissione Jacques Delors, ad esempio, hanno firmato nel novembre 1990 la Transatlantic Declaration USA-CE. Ciò ha dato la possibilità al Presidente della Commissione di incontrare due volte l'anno il Presidente degli Stati Uniti in regolari incontri al vertice. Similmente, la Declaration fra Comunità Europea e Giappone, firmata all'Aja nel luglio 1991, ha creato un quadro istituzionale attraverso il quale i Presidenti della Commissione e del Consiglio incontrano il Primo Ministro giapponese con cadenza annuale.

Questa risorsa può avere d'altro canto una valenza negativa qualora il Presidente della Commissione fallisca nel gestire efficacemente le questioni sul tappeto. Il prestigio di Delors, per esempio, fu seriamente danneggiato, all'interno ed all'esterno, allorché i Commissari MacSharry e Andriessen criticarono il Presidente per essere intervenuto a intralciare l'Uruguay Round delle trattative per il GATT nel 1992.

\section{Conclusione}

Il presente articolo ha avuto lo scopo principale di mettere in evidenza la struttura istituzionale che, a dispetto della con- 
trastata e malleabile natura della carica, si trova alla base della Presidenza della Commissione.

La discussione ha rivelato la presenza di almeno tre distinte e importanti forme della leadership del Presidente della Commissione. Una è quella relativa alla costruzione di un'amministrazione, o al suo miglioramento, ed è essenziale per fondare la base di un potere efficace e per esercitare la leadership in altri campi. La seconda forma della leadership è volta a definire compromessi e a costruire coalizioni, allo scopo di far funzionare senza intoppi la Comunità e di far progredire l'interesse comune alla costruzione comunitaria. La terza forma della leadership è relativa alla formazione e all'espansione dell'agenda politica, al fine di far avanzare il processo di integrazione.

Una importante domanda che ci si è posta era relativa alle risorse a disposizione del Presidente della Commissione nell'esercizio di queste forme di leadership. A questo proposito ci si è soffermati prima di tutto sui vincoli che il Presidente incontra nella sua azione. È possibile raggruppare tali vincoli in tre gruppi.

In primo luogo, la carica del Presidente della Commissione non è (e non vuole essere) fornita di legittimità democratica, manca dell'importante risorsa costituita dall'appoggio di un partito politico ed è caratterizzata dall'assenza di saldi legami con la società. È quindi difficile trovare elementi in comune fra la Presidenza della Commissione e le cariche di primo ministro o presidente a livello nazionale, con la possibile eccezione della Presidenza svizzera. Piuttosto, la Presidenza della Commissione è stata concepita e obbligata a servire gli interessi delle élites politiche nazionali - in particolare i capi dei governi degli stati membri - che hanno mantenuto uno stretto controllo sulla sua nomina.

In secondo luogo, la stessa posizione del Presidente all'interno della Commissione ne limita l'influenza e il margine d'intervento. La regola della collegialità, la mancanza del potere di nomina e revoca dei Commissari e di coloro che ricoprono altri posti-chiave nella Commissione, la frammentazione e l'eterogeneità dell'apparato della Commissione convergono in questa direzione.

In terzo luogo, è la logica istituzionale altamente policentrica della Comunità Europea a costituire il vincolo di maggiore portata al potere del Presidente. In un tale sistema, una leader- 
ship davvero prominente e dominante risulterebbe disfunzionale e potrebbe addirittura creare rischi di disgregazione della stessa Comunità. Le fonti di leadership devono pertanto essere disperse e il Presidente della Commissione non può che essere uno dei molti attori che si dividono la leadership.

Passando all'analisi delle risorse su cui il Presidente si basa per l'esercizio della sua leadership, si è messo in evidenza che, all'interno, la posizione di Presidente del Collegio, il fatto di avere il Gabinetto più esteso, la cooperazione con il Segretario Generale e il controllo su altri organi e canali lo aiutano a mobilitare il Collegio e l'apparato della Commissione e, quindi, a esercitare influenza al di fuori della Commissione.

In questo ambito, la membership nel Consiglio Europeo è l'atout fondamentale del Presidente sia rispetto all'esercizio della sua leadership mediatrice, sia rispetto alla possibilità di incidere sulla formazione dell'agenda politica della Comunità. Anche la Presidenza del Consiglio, il Segretariato del Consiglio e l'asse franco-tedesco possono essere trasformati in risorse esterne di potere, sempre che il Presidente riesca ad allearsi con tali attori. Infine, il Presidente può essere favorito (o ostacolato) nell'esercizio della leadership dalla sua posizione internazionale. Il successo nel mobilitare queste risorse esterne può, a sua vol$\mathrm{ta}$, facilitare una migliore utilizzazione delle sue risorse interne.

\section{[Traduzione di Giovanni Capoccia]}

\section{Riferimenti bibliografici}

Blondel, J. (1987), Political Leadership: Towards a general analysis, London, Sage.

- (1991), Commission de la Communauté et Gouvernements d'Europe Occidentale, in J. Blondel e L. Bardi (in collaborazione con G. Pasquino), cit.

Blondel, J e L. Bardi (in collaborazione con G. Pasquino) (1991), La Commission européenne: Processus de décision et le role des commissaires (Florence, novembre).

Blondel, J. e F. Müller-Rommel (a cura di) (1993), Governing Together: The Extent and Limits of Joint Decision Making in Western European Cabinets, Basingstoke, Macmillan.

Buchan, D. (1991), Man in the News: Jacques Delors, «Financial Times», 23-24 novembre, p. 8.

Bulletin of the European Communities (Bull EC) (1967), n. 4. 
Bulletin of the European Communities (1970), n. 1.

Bulletin of the European Communities (1972), n. 8.

Burgess, M. (1989), Federalism and European Union: Political Ideas, Influences and Strategies in the European Community. 1972-1986, London-New York, Routledge.

Cloos, J. et al. (1993), Le Traité de Maastricht: Génese, analyse, commentaires, Bruxelles, Établissements Émile Bruylant.

Commission des Communautés européennes (1993a), Secretariat général, SEC (93) 49/6, 4 febbraio.

- (1993b), SEC (93) 829, 25 maggio.

Conrad, Y. (1989), Jean Monnet et les Débats de la Fonction Publique Européenne: La Haute Autorité de la CECA (1952-1953), Louvainla-neuve, CIACO.

Coombes, D. (1970), Politics and Bureaucracy in the European Community: A Portrait of the Commission of the E.E.C., London, George Allen \& Unwin.

Dahrendorf, R. (1971a), Wieland Europa, in «Die Zeit», n. 28, 9 luglio, S. 3.

- (1971b), Ein neues Ziel für Europa, in «Die Zeit», n. 29, 16 luglio, S. 3.

Donnelly, M. e E. Ritchie (1994), The College of Commissioners and their Cabinets, in G. Edwards e D. Spense (a cura di), The European Commission, Harlow, Longman, pp. 31-61.

Edwards, G. e D. Spence (a cura di) (1994), The European Commission, Harlow, Longman.

Forsyth, M. (1981), Unions of States: The Theory and Practice of Confederation, Leicester, Leicester University Press.

Grant, C. (1994), Delors: Inside the House that Jacques Built, London, Nicholas Brealey.

Haas, E. (1958), The Uniting of Europe: Political, Social, and Economic Forces, 1950-1957, Stanford, CA, Stanford University Press.

Habermas, J. (1992), Citoyenneté et identité nationale: Réflexions sur l'avenir de l'Europe, in J. Lenoble e N. Dewandre (a cura di), L'Europe au soir du siècle: Identité et démocratie, Paris, Editions Esprit.

Hallstein, W. (1979), Die Europäische Gemeinschaft, Dusseldorf, Econ Verlag.

Hayward, J. (a cura di) (1993), De Gaulle to Mitterrand: Presidential Power in France, London, Hurst \& Company.

Hill, A. (1993), Summiteers at odds over Ecofin council's role, in «Financial Times», 13 dicembre, p. 2.

Howe, G. (1994), Conflict of Loyalty, London, Macmillan.

Hughes, C. (1962), The Parliament of Switzerland, London, Cassel \& Company Ltd.

Jacobs, F., R. Corbett e M. Shackleton (1992), The European Parliament, 2. ed., Harlow, Longman. 
Jenkins, R. (1989), European Diary 1977-1981, London, Colins.

Jones, G.W. (a cura di) (1991a), West European Prime Ministers, in «West European Politics», XIV, 2.

- (1991b), West European Prime Ministers in Perspective, in «West European Politics», XIV, 2, pp. 163-178.

King, A. (1975), Executives, in F.I. Greenstein e N.W. Polsby (a cura di), The Handbook of Political Science, Reading, MA, Addison Wesley, pp. 173-256.

Krenzler, H.G. (1974), Die Rolle der Kabinette in der Kommission des Europäischen Gemeinschaften, in «Europarecht», 2, pp. 59-67.

Le Monde (1972), 20 ottobre, p. 1.

Ludlow, P. (1982), The Making of the European Monetary System: A Case Study of the Politics of the European Community, London, Butterworth.

- (1991), The European Commission, in R.O. Keohane e S. Hoffmann (a cura di), The New European Community: Decision Making and Institutional Change, Boulder, Westview Press, pp. 85-132.

Marjolin, R. (1989), Architect of European Unity: Memoirs 1911-1986, London, Weidenfeld and Nicolson.

Milèsi, G. (1985), Jacques Delors, Paris, Pierre Belfond.

Morgan, R. (1992), Jean Monnet and the ECSC Administration: Challenges, Functions and the Inberitance of Ideas, in E.V. Heyek et al. (a cura di), Early European Community Administration, Baden-Baden, Nomos Verlagsgesellschaft, pp. 1-9.

Newhouse, J. (1968), Collision in Brussels, London, Faber.

Nöel, E. (1992), Témoignage: l'administration de la Communauté européenne dans la rétrospective d'un ancien baut fonctionnaire (interview par Roger Morgan), in E.V. Heyek et al. (a cura di), Early European Community Administration, Baden-Baden, Nomos Verlagsgesellschaft, pp. 145-158.

Nuallain, C.O. (a cura di) (1985), The Presidency of the Council of Ministers: Impact and Implications for National Governments, London, Croom Helm.

«Official Journal of the European Communities (OJ)» (1980), Vol. L, 261/16, 4 ottobre.

Règlement intérieure de la Commission (1963), in «Journal officiel des Communautés européennes», n. 17, 31 gennaio.

Ross, G. (1993), Sidling into Industrial Policy: Inside the European Commission, in «French Politics and Society», XI, 1, pp. 20-44.

- (1995), Jacques Delors and European Integration, Cambridge, Polity Press.

Sharp, M. (1990), Technology and the Dynamics of Integration, in W. Wallace (a cura di), The Dynamics of European Integration, London, Pinter, pp. 50-68.

Simonian, H. (1985), The Privileged Partnership: Franco-German Rela- 
tions in the European Community 1969-1984, Oxford, The Clarendon Press.

Smith, A. e M. Smyrl (1995), A la recherche d'interlocuteurs: La Commission européenne et le developpement territorial en France, in «Sciences et Société. Les cahiers du LERASS» (febbraio).

Spence, D. (1993), The European Community and German Unification, in C. Jeffery e R. Strum (a cura di), Federalism, Unification and European Integration, London, Frank Cass, pp. 136-163.

Spence, D. (1994), Structure, Functions and Procedures in the Commission, in G. Edwards e D. Spense (a cura di) (1994), pp. 101-103.

Taylor, P. (1993), International Organization in the Modern World: The Regional and Global Process, London, Pinter.

Wallace, H. (1983), Negotiation, Conflict, and Compromise: The Elusive Pursuit of Common Policies, in $\mathrm{H}$. Wallace, W. Wallace e C. Webb (a cura di), Policy Making in the European Community, 2. ed., Chichester, John Wiley \& Sons, pp. 43-80.

Wallace, H. e G. Edwards (1976), European Community: The Evolving Role of the Presidency of the Council, in «International Affairs», LII, 4, pp. 535-550.

Wallace, W. (1983), Less than a Federation, More than a Regime: the Community as a Political System, in H. Wallace, W. Wallace e C. Webb (a cura di), Policy Making in the European Community, 2. ed., Chichester, John Wiley \& Sons, pp. 403-436.

- (1993), Walter Hallstein from the British Perspective, paper presentato all'Institut fur Europaische Politik, Bonn (settembre).

Weiler, J.H.H. (1992), After Maastricht: Community Legitimacy in Post-1992 Europe, in W.J. Adams (a cura di), Singular Europe: Economy and Polity of the European Community after 1992, Michigan, University of Michigan Press, pp. 11-41.

Werts, J. (1992), The European Council, Amsterdam, North-Holland.

Wright, V. (1989), The Government and Politics of France, London, Unwin-Heyman, $3^{\mathrm{a}} \mathrm{ed}$. 